\title{
INVESTIGATION OF HOMOCYSTINURIA-ASSOCIATED MUTATIONS IN THE ACTIVE SITE OF CYSTATHIONINE $\beta$-SYNTHASE
}

By

Duale Ahmed, B.Sc.H.

A thesis submitted to the Faculty of Graduate and Postdoctoral Affairs in partial

fulfilment of the requirements for the degree of

Master of Science

in

Biology

Department of Biology

Ottawa-Carleton Institute of Biology

Carleton University

Ottawa, Ontario

September 2014

(C)2014

Duale Ahmed 


\section{ABSTRACT}

Homocystinuria is an inherited autosomal recessive disorder that affects an individual's ability to metabolize the L-methionine derivative L-homocysteine. Cystathionine $\beta$-synthase (CBS) catalyzes the condensation of L-homocysteine and Lserine in the first step of the reverse transsulfuration pathway, which shunts excess Lhomocysteine to L-cysteine biosynthesis. More than 100 mutations of the CBS gene have been associated with homocystinuria. Treatment for this disease includes supplementation with vitamin $\mathrm{B}_{6}$ and/or betaine and restriction of dietary L-methionine. Refinement of these therapeutic regimes will be enabled by an enhanced understanding of the distinct effects of homocystinuria-associated mutations as well as detailed characterization of the roles of the residues and hydrogen bonding networks in the enzyme active site. This study: (1) probed the role of active site residues D319, S323 and Y324 in the positioning of the pyridoxal 5'-phosphate cofactor of the model yeast CBS enzyme and (2) investigated a set of 11 homocystinuria-associated mutations in the context of human CBS. In the first study, substitution of D319 with alanine, removing only the carboxylate moiety of the side chain, eliminated activity, while the S323A and Y324F variants, each lacking the side chain hydroxyl group, had negligible effect on the kinetic parameters, compared to the wild-type enzyme. Residue G148 provides the mobile loop the flexibility to transition between the open and closed active site conformations. Residues E176, K384 and G307 are involved in maintaining a catalytically productive active site architecture. Residues T257 and G259 serve critical roles in PLP binding and in the bi-directional communication between the heme and PLP 
cofactors. Residues G305 and S349 also participate in positioning of the PLP cofactor. Understanding the structure-function relationships that underlie the molecular basis for each distinct homocystinuria-associated mutation of CBS provides insight that can be used to refine treatment for patients with homocystinuria. 


\section{ACKNOWLEDGEMENTS}

Dr. Martin Luther King Jr. once said, "The ultimate measure of a man is not where he stands in moments of comfort and convenience, but where he stands at times of challenge and controversy". The challenge of completing my M.Sc. degree would not have been possible without the rays of light that have guided me through this tunnel.

Firstly, I would like to thank Dr. Susan M. Aitken for giving me the opportunity to work in her lab as an undergraduate albeit my grades signified that I did not deserve nor earned that opportunity. Yet, you helped me realized that research is my passion. Without your insight, I would not be in the position that I am in right now. I still have a lot to learn when it comes to being a researcher but you have helped me get this far and I know you will continue to guide me to becoming the researcher I want to be during my tenure as a Ph.D. candidate in your lab.

I would like to extend my appreciation to my committee members, Dr. Amanda J. MacFarlane and Dr. Kenneth B. Storey. Your insights and feedback were essential to the creation of this thesis and I am sincerely thankful for your guidance!

I would not have gotten to this point without those who have already paved the road ahead for me. A special thank you goes to Allison Jaworski, Dr. Dominique Morneau and Edgar Abouassaf. You have been with me since Day 1 in the Aitken Lab and I would not be the researcher that I am today without your expertise. To Ally, thank you for vouching for me to Sue when it came to taking on a fourth year honour student even though my grades did not warrant you going out on a limb for me. Your guidance as my TA, 4908 graduate supervisor, friend and an overall awesome person is something that cannot be replaced and for that, I am forever grateful. To Dom, working in the lab 
was never a dull moment when you were around. You always helped me come up with ideas when I wasn't able to do on my own and while your taste in comic book heroes may be questionable, your friendship never was. To Eddy (and past Eddy), thank you for showing me the ropes when it comes to working on the CBS enzyme. You helped me become a better TA and RA. For all the gains I have made as a person, I thank you. My fellow graduate students, Emily Hopwood, Sorin Gustin and Victoria Samaki, were always willing to help me out when I needed it and helped create a great working environment in the lab. I also extend my gratitude to the undergraduate students that I had the pleasure of working with during my time in the lab, Usman Khan, Katrina Farrell and Menachem Loewenthal. You have helped me with my research and you have taught me something even though it was my job to teach you. I will always cherish the friendships I have made with my fellow graduate students within the department, Mohsen Hooshyar, Magdalena Bruno, Bahram Samanfar, Katayoun Omidi, Kristina Shostak and Dan Burnside. All of you have made my time here an experience that I will never forget! Lastly but certainly not least, I would not be the person I am today without the support of my family. Hooyo and Aabo, you brought me into this world, nurtured me and raised me to become someone who believes that I can accomplished anything that I sent my mind to do. While life was not always easy for us, you still gave everything you had to make sure that my three brothers and I never felt those hardships. Hopefully, I have and will continue to live up to the name you have bestowed onto me. To the rest of my support system, my brothers Mohamed, Mubarak and Mukhtar; my cousin Kombe; my aunt Muna; my brother from another mother Hussein; thank you for being there for me whenever I needed you. The members of my family that I have not mentioned, I am 
grateful for you as I would be here without you. You all are my pillars and without you I would crumble.

I know that I have accomplished a great deal thus far but there is still a ways to go before I have reached my potential. All of you have played a role in getting me to where I am today. I am excited for what the future holds for me knowing that I have all of you to lean on for support. Malcolm X once said, "The future belongs to those who prepare for it today". The one thing I know is that I am ready for what is next as long as you all have my back! 
TABLE OF CONTENTS

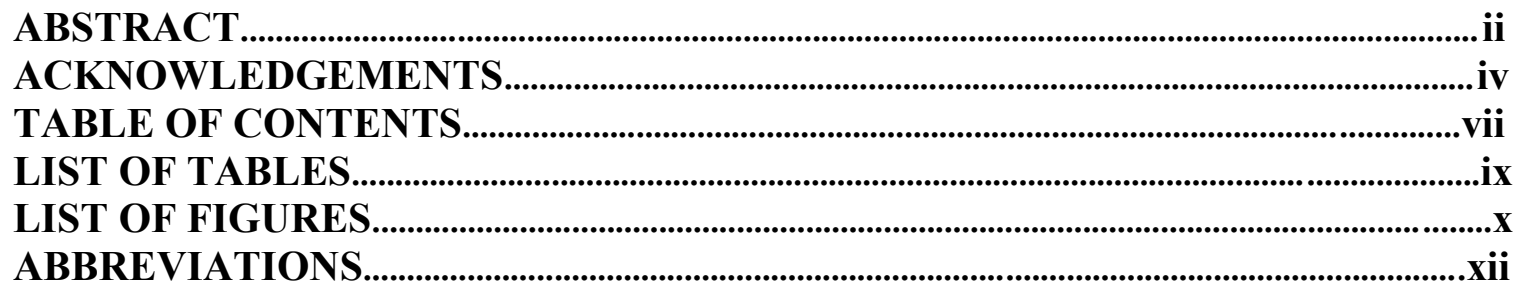

1. INTRODUCTION ............................................................................................................

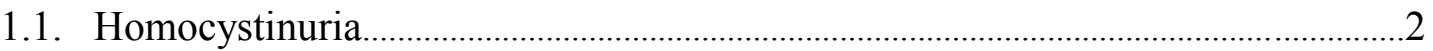

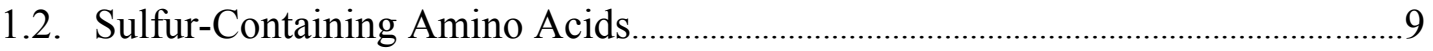

1.3. The Transsulfuration and Reverse Transsulfuration Pathways.............................10

1.3.1. Pyridoxal 5'-Phosphate ................................................................................13

1.4. Cystathionine $\beta$-synthase ................................................................................................

1.4.1. Protein Architecture...............................................................................................

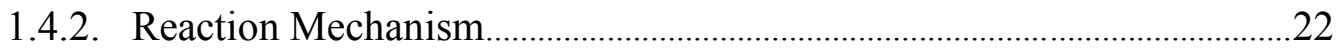

1.4.3. Role of CBS in Hydrogen Sulfide Production..............................................2

1.4.4. Proposed Roles of Heme in hCBS.............................................................28

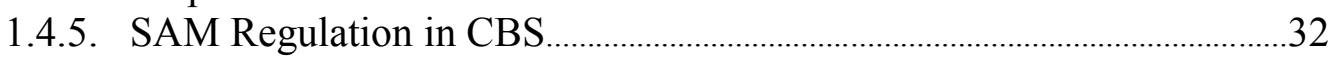

1.4.6. Homocystinuria-associated Mutations of CBS..............................................33

1.5. Focus of Study and Objectives.........................................................................................

2. METHODS...............................................................................................................................37

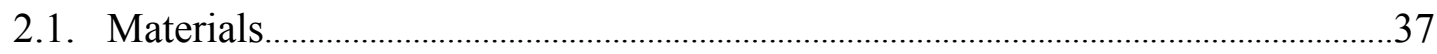

2.2. Construction of Site-Directed Mutants...........................................................................37

2.3. Expression and Purification of yfCBS Mutants...………………………………......39

2.4. Expression and Purification of htCBS Mutants........................................................40

2.5. Spectroscopic Characterization of htCBS...............................................................40

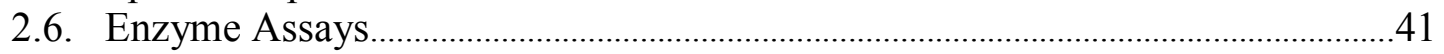

2.6.1. Hydrolysis of L-Cystathionine by yfCBS ………………………………......4 41

2.6.2. Condensation of L-Serine and L-Homocysteine by htCBS.......................43

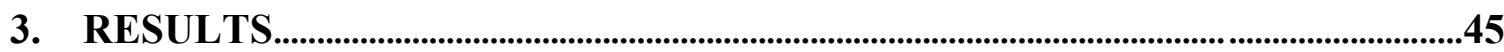

3.1. The Construction and Purification of the Site-Directed Variants..........................45

3.2. Kinetic Characterization of the yfCBS Variants.......................................................45

3.3. Kinetic Characterization of the htCBS Variants......................................................50

3.4. Spectral Characterization of the htCBS Wild-Type Enzyme and Site-Directed Variants. 


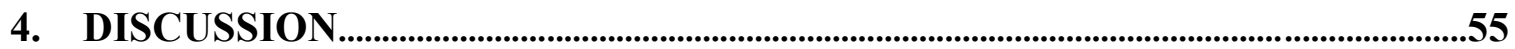

4.1. The yfCBS Variants (D319A, S323A \& Y324F) ....................................................55

4.2. The Truncated Human CBS Enzyme...........................................................................59

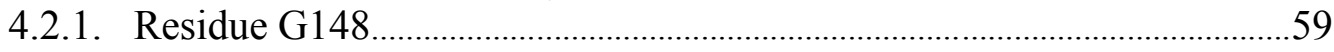

4.2.2. Residues E176 and K384 ........................................................................62

4.2.3. Residues T257 and G259......................................................................65

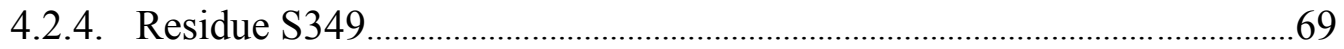

4.2.5. Residues G305 and G307 ………………………………………………......

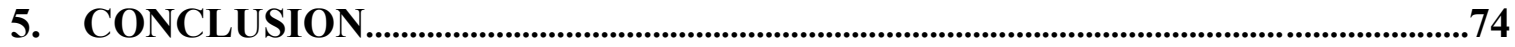

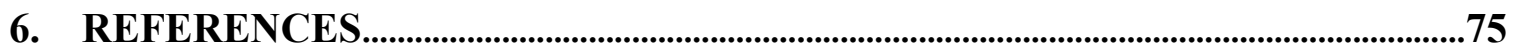




\section{LIST OF TABLES}

Table 2.1. $\quad$ Primers used in the construction of the yfCBS and htCBS site-directed mutants.

Table 3.1. Protein yield of yfCBS and htCBS variants...................................................49

Table 3.2. Steady-state kinetics parameters of wild-type yfCBS and the D319A, S323A and Y324F variants for the reverse-physiological hydrolysis of Lcystathionine.

Table 3.3. Steady-state kinetic parameters for the condensation of L-serine (L-Ser) and L-homocysteine (L-Hcys) by wild-type htCBS and homocystinuriaassociated variants. 


\section{LIST OF FIGURES}

Figure 1.1. The major pathways of sulfur amino acid metabolism.......................................

Figure 1.2. The transsulfuration pathway of bacteria and plants, the reverse transsulfuration pathway of mammals and yeast as well as their association to the methionine cycle

Figure 1.3. The variety of reactions catalyzed by PLP-dependent enzymes .15

Figure 1.4. A comparison of the organization of domains among a selection of representative fold-type II PLP-dependent enzymes. 16

Figure 1.5. Structural differences in the positioning of the regulatory domain in the hCBS ( $\Delta 516-525)$ and dCBS protein structures. 21

Figure 1.6. Reaction mechanism of CBS, showing the PLP-bound intermediates as well as their associated absorption maxima, as observed in yCBS..............23

Figure 1.7. The four known $\beta$-replacement reactions catalyzed by CBS........................27

Figure 1.8. Enolimine and ketoenamine tautomers of the PLP-internal aldimine in human cystathionine $\beta$-synthase...........................................................................

Figure 1.9. Proposed mechanism for the heme-CO linked inactivation of hCBS.........31

Figure 1.10. A 3D model of the hCBS $\triangle 516-525$ variant crystal structure showing both CBS domains (CBS1 and CBS2) and the location of the proposed binding sites of SAM (S1 and S2). 34

Figure 2.1. DNTB assay utilized to measure the reverse-physiological hydrolysis of Lcystathionine by CBS.

Figure 2.2. The assay employed to determine the $\beta$-replacement activity of CBS, via the use of CBL and LDH coupling enzymes. 44

Figure 3.1. Agarose gel of PCR amplicons produced for the construction of the htCBS site-directed mutants via overlap-extension PCR 46

Figure 3.2. A SDS-PAGE gel, stained with Comassie Blue, showing $4 \mu \mathrm{g}$ each of the wild-type and S323A, Y324F and D319A site-directed variants of yfCBS.

Figure 3.3. $\quad$ A SDS-PAGE gel stained with Comassie Blue reagent showing $4 \mu \mathrm{g}$ of the wild-type and site-directed variants of htCBS. 48

Figure 3.4. UV-Visible spectra of the wild-type htCBS (black) and ytCBS (red) enzymes 53

Figure 3.5. UV-Visible spectra of the purified htCBS variants examined in this study. .54 
Figure 4.1. Proposed active site contacts in the yCBS-aminoacrylate complex............58

Figure 4.2. The placement of residue G148 (red) within the CBS active site and its direct and indirect interaction with T146 (pale blue) and the external aldimine (E-Ser; Yellow), respectively.... 61

Figure 4.3. The location of the K177 hydrogen bonding network to which K384 and E176 are members of said group. 64

Figure 4.4. A view of the glycine-rich loop that positions the PLP cofactor via interactions with the phosphate moiety. .66

Figure 4.5. Location of key residues and $\alpha$-helix 8, which extends from the heme cavity to the PLP-binding site, proposed to be involved in bi-directional communication between the two cofactors....................................................68

Figure 4.6. The interaction between residue S349 (red) of hCBS and the N1 position of the PLP cofactor (E-Ser; yellow) ....................................................... 70

Figure 4.7. Position of G305 and G307 (red) at the mouth of the hCBS active site.....73 


\section{ABBREVIATIONS}

AD

反-ALA

ATP

BHMT

cAMP

CBL

CBS

CGL

CGS

CNS

CO

CT

D,L-Hcys

dCBS

DNA

DTNB

DTT
Alzheimer disease

$\delta$-aminolevulinic acid

Adenosine triphosphate

Betaine:homocysteine methyltransferase

Cyclic adenosine monophosphate

Cystathionine $\beta$-lyase

Cystathionine $\beta$-synthase

Cystathionine $\gamma$-lyase

Cystathionine $\gamma$-synthase

Central nervous system

Carbon monoxide

Computed tomography

D,L-Homocysteine

Drosophila melanogaster cystathionine $\beta$-synthase

Deoxyribonucleic acid

5,5'-Dithiobis-(2-nitrobenzoic acid)

Dithiothreitol 


\begin{tabular}{|c|c|}
\hline E. coli & Escherichia coli \\
\hline E-Cth & External aldimine of L-cystathionine \\
\hline E-Ser & External aldimine of L-serine \\
\hline E.C. & Enzyme Commission \\
\hline eCBL & Escherichia coli cystathionine $\beta$-lyase \\
\hline EDTA & Ethylenediaminetetraacetic acid \\
\hline $\mathrm{Fe}^{3+}$ & Ferric Iron State \\
\hline $\mathrm{Fe}^{2+}$ & Ferrous Iron State \\
\hline GSH & Glutathione \\
\hline $\mathbf{H}_{2} \mathbf{S}$ & Hydrogen sulfide \\
\hline hCBS & Human cystathionine $\beta$-synthase \\
\hline htCBS & Human truncated cystathionine $\beta$-synthase \\
\hline IPTG & Isopropyl- $\beta$ - $D$-thiogalactopyranoside \\
\hline ITC & Isothermal titration calorimetry \\
\hline L-Cth & L-Cystathionine \\
\hline L-Cys & L-Cysteine \\
\hline L-Hcys & L-Homocysteine \\
\hline
\end{tabular}




$\begin{array}{ll}\text { L-Met } & \text { L-Methionine } \\ \text { L-Ser } & \text { L-Serine } \\ \text { LB } & \text { Luria Broth } \\ \text { LDH } & \text { L-lactate dehydrogenase } \\ \text { NADH } & \text { Nicotinamide adenine dinucleotide, reduced form } \\ \text { NaCl } & \text { Sodium Chloride } \\ \text { NH } & \text { Ammonia } \\ \text { Ni-NTA } & \text { Nickel-nitrilo triacetic acid } \\ \text { NMDA } & N \text {-methyl-D-Aspartate } \\ \text { PLP } & \text { Pyridoxal 5'-phosphate } \\ \text { NO } & \text { Nitric oxide } \\ \text { OD } & \text { Optical density } \\ \text { MTHFr } & \text { Methionine synthase } \\ & \end{array}$


RNA

SAH

SAHH

SAM

SAMS

SDS-PAGE

TD

THF

TNB $^{-}$

TrpS

TS

UV

yCBS

yfCBS

ytCBS
Ribonucleic acid

S-adenosylhomocysteine

$S$-adenosylhomocysteine hydrolase

$S$-adenosylmethionine

$S$-adenosylmethionine synthetase

Sodium dodecyl sulfate-polyacrylamide gel electrophoresis

Threonine deaminase

Tetrahydrofolate

3-thio-6-nitrobenzoate

Tryptophan synthase

Threonine synthase

Ultraviolet

Yeast cystathionine $\beta$-synthase

Yeast full-length cystathionine $\beta$-synthase

Yeast truncated cystathionine $\beta$-synthase 


\section{INTRODUCTION}

Inborn errors of metabolism, as first described by Archibald Garrod in 1908, are a class of disorders in which genetic defects affect the function of the encoded enzymes (Scriver, 2008). The presentation of symptoms is commonly correlated with the accumulation of metabolite(s), resulting from the reduced function of the associated enzyme, which impedes cellular and/or physiological processes (Pasternak, 2005). Treatment of these disorders is complicated by the ability of distinct mutations of a gene to result in clinical manifestations which may vary in presentation and/or severity and with respect to treatment options, depending on the specific allele(s) an individual possesses. This presents researchers with the challenge of understanding the structurefunction relationships that underlie each distinct disease-associated allele and how these are manifested in vivo, as well as clinically. This study explores these structure-function relationships, focusing on homocystinuria-associated mutations and hydrogen bonding networks in the active site of the enzyme cystathionine $\beta$-synthase.

Cystathionine $\beta$-synthase (CBS) catalyzes the condensation of L-serine (L-Ser) and L-homocysteine (L-Hcys), producing L-cystathionine (L-Cth), in first committed step of the reverse transsulfuration pathway, which transfers the sulfur atom of L-Hcys, a product of L-methionine (L-Met) metabolism, to L-cysteine (L-Cys) (Figure 1.1) (Stipanuk, 2004). Typical of enzymes catalyzing transformations of amino acid substrates, CBS is reliant on the catalytically versatile pyridoxal 5'-phosphate (PLP) cofactor. Higher eukaryotic forms of CBS (e.g. Drosophila melanogaster and human, but not yeast) bind heme in addition to PLP (Kery et al., 1994). Mutations in the gene encoding CBS are the primary cause of homocystinuria, a condition characterized by high levels of L-Hcys and L-Met in the plasma and urine (Mudd, et al., 1985). Worldwide 
prevalence of homocystinuria, the most common of sulfur amino acid metabolic disorders, is 1 in 344,000 births but recent reports suggest the incidence rate may be higher (Mudd et al., 2001; Yap, 2012). An enhanced understanding of the effect of homocystinuria-associated mutations on the biochemical properties of CBS will aid in the refinement of therapeutic approaches for patients with homocystinuria.

\subsection{Homocystinuria}

Homocystinuria was first described in 1962 by independent groups in Northern Ireland and in the United States examining the amino acid content of the urine of children with mental retardation, and was linked to CBS deficiency in 1964 (Carson et al., 1962; Gerritsen et al., 1962; Mudd et al., 1964). It is biochemically characterized by elevated levels of plasma and urine L-Hcys $(>100 \mu \mathrm{M})$ and L-Met. Prevalence of homocystinuria varies from 1:65,000 in Northern Ireland to 1:900,000 in Japan (Mudd et al., 2001; Naughten et al., 1998). Recent reports suggest the incidence rate may be higher as genetic screening of specific CBS mutations has resulted in estimates ranging from 1:6,500 to 1:20,000 in European countries, including Denmark, Germany, Norway and the Czech Republic, and a study in Qatar reported an incidence rate of 1:1200 based on the biochemical screening of newborns (Yap, 2012; Kozich et al., 2010; Zschocke et al., 2009). 


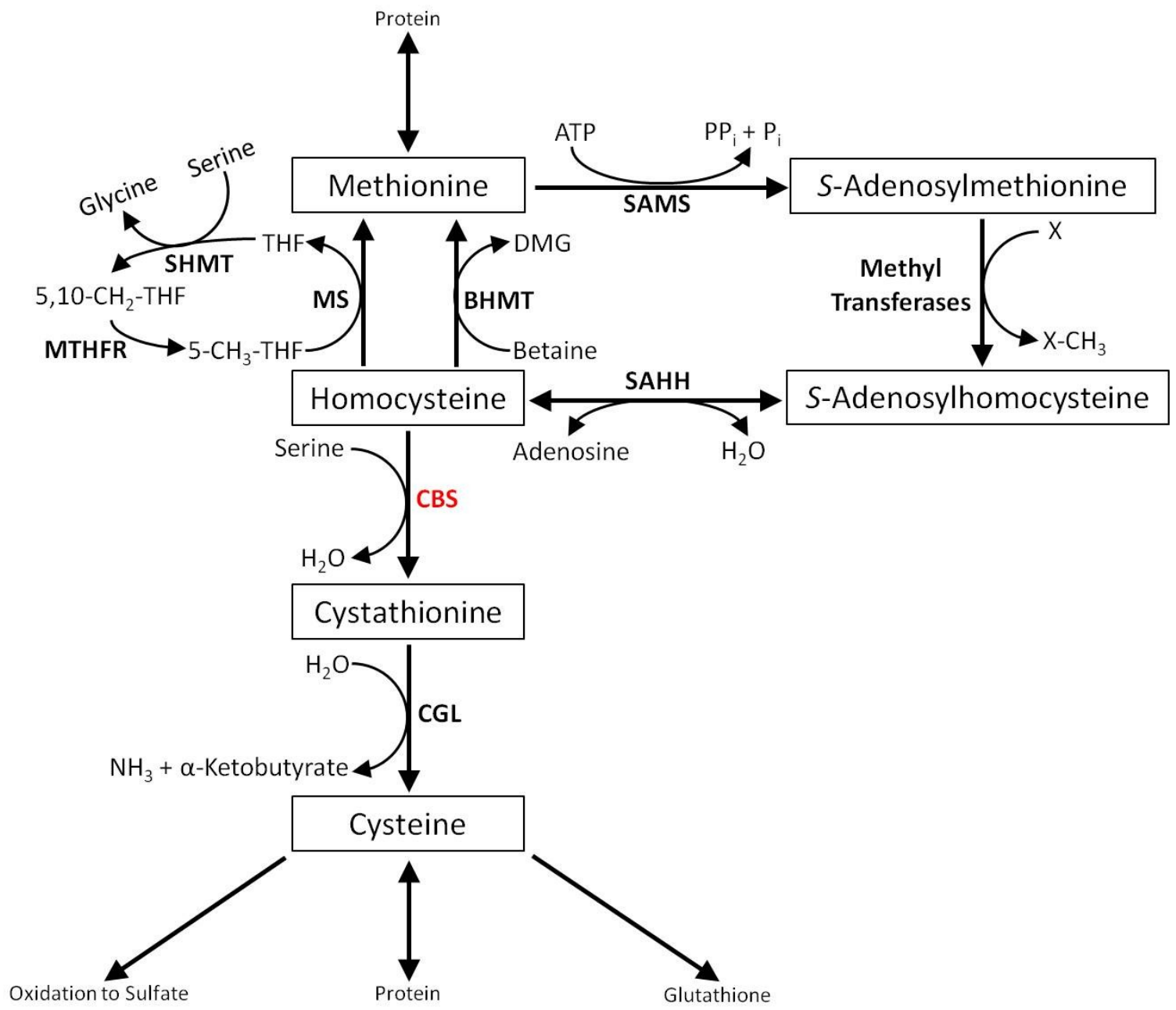

Figure 1.1: The major pathways of human sulfur amino acid metabolism (adapted from Brosnan \& Brosnan, 2006). SAMS: $S$-adenosylmethionine synthetase, ATP: Adenosine triphosphate, $\mathrm{PP}_{\mathrm{i}}$ : Pyrophosphate, $\mathrm{P}_{\mathrm{i}}$ : Inorganic phosphate, $\mathrm{SAHH}: S$ -

adenosylhomocysteine hydrolyze, BHMT: Betaine:homocysteine methyltransferase, DMG: Dimethylglycine, MS: Methionine synthase, THF: Tetrahydrofolate, SHMT: Serine hydroxymethyltransferase, 5,10- $\mathrm{CH}_{2}$-THF: 5,10-methylene-THF, MTHFR: 5,10methylene-THF reductase, 5- $\mathrm{CH}_{3}$-THF: 5-methyl-THF, CBS: Cystathionine $\beta$-synthase, CGL: Cystathionine $\gamma$-lyase. 
Homocystinuria resulting from CBS enzyme deficiency is inherited as an autosomal recessive trait. The $C B S$ gene, which encodes the enzyme, is located on the $21^{\text {st }}$ human chromosome (Munke et al., 1988). The severity of the observed symptoms ranges from nearsightedness and limited bone demineralization, to blockage of blood vessels due to thromboembolisms, and their severity is dependent on a number of factors including the individual's environment, diet, and the specific CBS mutation (Mudd et al., 2001). As of 2011, 164 distinct CBS mutations associated with the disorder have been reported (http://cbs.lf1.cuni.cz/mutations.php). Homocystinuria-associated mutations can be categorized according to patients' responsiveness to therapeutic doses of the PLP precursor, pyridoxine (vitamin $\mathrm{B}_{6}$ ), which may be linked to the degree of reduced PLP affinity associated with each diseased CBS enzyme. Factors affecting the enzyme's affinity $\left(K_{d}\right)$ for PLP include enzyme misfolding and alterations in active site architecture (Miles and Kraus, 2004). Elevated in vivo PLP concentration over the $K_{d}$ of a particular variant can allow for proper enzyme formation and designation as a pyridoxine responsive variant.

The A144V, R266K, I278T, R336H, K384E or L539S variants have been shown to be among the $50 \%$ of variants that are responsive to pyridoxine treatment in vivo. Conversely, patients with the R121L, R125Q, C165Y, E176K, T191M, T257M, T262M or G307S mutations have been shown to be nonresponsive to pyridoxine treatment (Mudd et al., 2001). Although the majority of the mutations are identified as private missense mutations, single base substitutions found in one or a small number of individuals, more than half $(51 \%)$ of homocystinuria diagnoses correspond to four 
prevalent variants of CBS: I278T, T191M, G307S and R336C, which all but the I278T substitution are nonresponsive to pyridoxine (Kraus et al., 1999; Urreizti et al., 2003).

The clinical presentation of homocystinuria is primarily associated with four organ systems: the vascular system, the central nervous system (CNS), the eye and the skeletal system. The most frequent cause of death in patients with CBS deficiency is the development of vascular thromboembolisms, which have been reported to develop in patients as young as 19 months (Kerrin et al., 1996). An international study conducted by Mudd and colleagues (1985) demonstrated that thromboembolic development is not restricted to a particular section of the vascular system. In this study, 253 distinct thromboembolic events were reported for 158 of the 629 patients examined. Of these, $51 \%$ involved the peripheral veins while $32 \%$ were found to be cerebrovascular, $11 \%$ occurred in the peripheral arteries, and $4 \%$ were associated with myocardial infarctions (Mudd et al., 1985). Several hypotheses attempting to explain the association between elevated plasma L-Hcys and increased prevalence of thromboembolic events have been put forward. For example, Majors and colleagues (2002) provided evidence that L-Hcys binding to fibronectin, a cysteine-rich protein involved in cell migration and wound healing, may result in abnormal thrombosis formation by impairing fibronectin-fibrin binding. Other reported hypotheses suggest that attacks by the metabolite's cyclic thioester form, L-Hcys thiolactone, on nucleophilic amino acid side chains of proteins lining the vascular endothelium or reactive oxygen species produced by the reduced and cyclic forms of L-Hcys may damage the vascular endothelium resulting in the development of thromboembolisms (Perna et al., 2003; Stamler and Slivka, 1996; Jakubowski, 2008). However, while these in vitro studies assumed a causal role of L- 
Hcys in the development of vascular occlusions, recent clinical studies suggest the relationship may be correlational.

The presence of hyperhomocysteinaemia, generally described as total L-Hcys levels greater than $15 \mu \mathrm{M}$, independent of any genetic disorder were proposed to be an independent risk factor for vascular diseases (Clarke et al., 1991; Saposnik et al., 2009). However, studies have revealed that lowering the total L-Hcys concentration via Bvitamin supplementation does not decrease the risk of vascular disease. A large study analyzing the stroke outcomes of 5522 adults diagnosed with cardiovascular disease were given folic acid, vitamins $\mathrm{B}_{6}$ and $\mathrm{B}_{12}$ (cobalamin), or a matching placebo for an average of 5 years, found that B-vitamin supplementation lowered total L-Hcys concentration, but it did not reduce the risk of major vascular events (Bonaa et al., 2006). Zhang et al. (2013) conducted a meta-analysis of 18 randomized controlled trials consisting of 57,143 individuals and found that the combination of folic acid and Vitamins $\mathrm{B}_{6}$ and $\mathrm{B}_{12}$ was not associated with a significant reduction in stroke risk. As a result, there is some uncertainty about the role of L-Hcys, whether causal or incidental, in vascular disease.

Neurological abnormalities such as seizures and mental retardation are often the first recognized sign of CBS deficiency (Mudd et al., 1985). Neurological disorders such as neural tube defects can be attributed to L-Hcys, acting as an $N$-methyl-D-aspartate (NMDA) receptor agonist, disrupting neural tube development through an influx of calcium ions and reactive oxygen species, resulting in neuronal damage (Lipton et al., 1997). Studies have also suggested a link between elevated plasma L-Hcys levels and Alzheimer disease (AD). For example, Clarke et al. (1998) found that plasma L-Hcys levels were significantly higher in patients with AD than the controls. Among patients 
with $\mathrm{AD}$, the study observed a higher degree of disease progression over a 3-year period, as determined by computed tomography (CT) scans, in patients with a higher total plasma L-Hcys concentration (Clarke et al., 1998). A recent study found that L-Hcys exacerbates the three major pathological features of $\mathrm{AD}$ in a mouse model: memory loss, and $\beta$ amyloid and tau protein accumulation (Li et al., 2014).

A consistent observation in patients with homocystinuria is the appearance of ectopia lentis, the dislocation of the ocular lens due to a disruption of the zonular fibres, which are responsible for maintaining the lens' position within the eye (Mudd et al., 2001; Cruysberg et al., 1996). It is believed that excess L-Hcys in the aqueous humour forms disulfide bonds with the L-Cys residues of the glycoprotein fibrillin, which plays a key role in maintaining the strength and elasticity of the ocular fibers, thus compromising its structural integrity (Sakai, 1990; Mudd et al., 2001). The resulting loosening of the ocular lens leads to the development of myopia (nearsightedness) (Mudd et al., 1985).

The development of osteoporosis (decrease in bone mass and density) in the spine and the long bones (femur, tibia and humerus) is the most common skeletal change found in patients with homocystinuria. At least $50 \%$ of patients are reported to have varying degrees of osteoporosis by the age of 20 , with earlier onset in those with the pyridoxinenonresponsive disease variants such as the E176K, T257M and G307S variants (Mudd et al., 1985). It has been suggested that excess L-Hcys results in defective crosslinking of collagen within the bone (Harris and Sjoerdsma, 1966). The crosslinking of collagen molecules involves the formation of aldehyde groups via the oxidation of terminal amino groups of lysine or hydroxyllysine residues. L-homocysteine is capable of reacting with the exposed aldehyde groups of immature collagen molecules, resulting in the formation 
of thiazine rings that subsequently reduce the structural integrity of the bone (Jackson, 1973; Kang and Trelsand, 1973). Taken together, these findings demonstrate the importance of understanding the role L-Hcys plays in the pathophysiology of homocystinuria is critical to finding new therapeutic approaches.

Three different modes of treatment are currently available to patients with homocystinuria. The treatment of choice consists of high doses of pyridoxine. In order to clinically determine a patient's responsiveness to pyridoxine, physicians conduct the pyridoxine challenge test, consisting of an initial daily intake of $100 \mathrm{mg}$ pyridoxine followed by an increase in dosage to $200 \mathrm{mg}$ and then $500 \mathrm{mg}$, if a $30 \%$ reduction in the total plasma L-Hcys is not observed. If no significant change in plasma L-Hcys is observed, the patient is considered $\mathrm{B}_{6}$-non-responsive (Pagon et al., 2011). Methionine synthase (MS) activity is dependent on cobalamin while the folate cycle provides the methyl moiety transferred to L-Hcys by methionine synthase (Figure 1.1) (Morrow and Barness, 1972). Therefore, folic acid and cobalamin are added to supplement pyridoxine treatment to prevent their depletion in the remethylation of L-Hcys (Morrow and Barness, 1972). A methionine-restricted/cysteine-supplemented diet which includes a combination of natural and synthetic proteins, as well as fruits and vegetables can be used alone or in conjunction with other treatments of CBS deficiency (Yap, 2012). Supplementation with betaine provides an alternative treatment and is chiefly employed for pyridoxinenonresponsive patients and in cases where dietary compliance is an issue (Singh et al., 2004). Betaine serves as a methyl donor for betaine:homocysteine methyltransferase (BHMT) in the remethylation of L-Hcys observed primarily in liver and kidney tissues (Singh et al., 2004). 


\subsection{Sulfur-Containing Amino Acids}

The presence of excess L-Hcys in plasma and urine is an indicator of an imbalance in the metabolism of sulfur-containing amino acids L-Met and L-Cys. This has implications for cellular homeostasis because, in addition to their roles in the formation of proteins, L-Met and L-Cys are precursors of metabolites central to processes including maintenance of the redox status and methyl transfer reactions ( $\mathrm{Wu}, 2009)$. Cysteine is the limiting amino acid for the synthesis of glutathione (GSH), key to cellular redox regulation via the scavenging of free radicals and other reactive oxygen species (Wu et al., 2004; Banerjee and Zou, 2005; Carballal et al., 2013). The methionine cycle begins with the transfer of an adenosyl moiety, from ATP, to L-methionine by Sadenosylmethionine synthetase (SAMS) to create $S$-adenosylmethionine (SAM) (Brosnan and Brosnan, 2006). SAM is the primary methyl donor in the biosynthesis of compounds including DNA, RNA, small molecules and amino acids (Finkelstein and Martin, 1984; Stipanuk, 2004; Brosnan and Brosnan, 2006). Transfer of the methyl group of SAM to the various methyl acceptors results in the formation of $S$-adenosylhomocysteine (SAH), which is subsequently hydrolyzed to L-Hcys and adenosine by SAH-hydrolase (SAHH) (Figure 1.1). L-homocysteine is situated at a crucial metabolic branch point between LHcys remethylation, either by MS or BHMT, and the reverse transsulfuration pathway in which the sequential action of the enzymes CBS and cystathionine $\gamma$-lyase (CGL) convert L-Hcys to L-Cys (Stipanuk, 2004) (Figure 1.1). 


\subsection{The Transsulfuration and Reverse Transsulfuration Pathways}

The transsulfuration and reverse transsulfuration pathways interconvert L-Cys and L-Hcys, with the direction of the pathway (L-Cys to L-Hcys or vice versa) dependent on the evolutionary lineage of the organism (Figure 1.2). Plants and microorganisms have the ability to reduce inorganic sulfur for the de novo production of sulfur-containing amino acids, while animals cannot and must obtain L-Met through in their diet (Atmaca, 2004; Aitken and Kirsch, 2005). L-methionine is an essential amino acid but L-Cys, which is produced from L-Met, is considered a conditionally essential amino acid since it can derive either from diet or from the breakdown of L-Met (Stipanuk, 2004; Wu, 2009).

The transsulfuration pathway of bacteria and plants converts L-Cys to L-Hcys by the action of cystathionine $\gamma$-synthase (CGS) and cystathionine $\beta$-lyase (CBL) (Steegborn et al., 1999). Subsequent methylation of L-Hcys by MS yields L-Met (Figure 1.2). In mammals and yeast, the reverse transsulfuration pathway serves as the only catabolic route for the removal of excess L-Hcys, a product of the methionine cycle (Yadav and Banerjee, 2012; Smith et al., 2012). CBS catalyzes the condensation of L-Hcys and L-Ser, forming L-Cth, subsequently hydrolyzed by CGL, producing L-Cys, $\alpha$-ketobutyrate and ammonia $\left(\mathrm{NH}_{3}\right)$ (Figure 1.2) (Janosik et al., 2001a; Banerjee et al., 2003).

Comparison of the $K_{m}$ values of the competing branch-point enzymes metabolizing L-Hcys demonstrates that the remethylation of L-Hcys is favoured over transsulfuration, as MS and BHMT have a higher affinity for L-Hcys $\left(K_{m}(\mathrm{MS})=0.06\right.$ $\left.\mathrm{mM} ; K_{m}(\mathrm{BHMT})=0.012-0.06 \mathrm{mM}\right)$ than $\mathrm{CBS}\left(K_{m}=0.17 \mathrm{mM}\right)$ (Finkelstein, 1998; Janosik et al., 2001a). As the concentration of L-Hcys exceeds the capacity of MS and BHMT, and approaches the $K_{m}$ of CBS, flux through the reverse transsulfuration pathway 
is increased, thereby shunting excess L-Hcys irreversibly to L-Cys biosynthesis. This shift is enhanced by the presence of a concentration of SAM sufficient for cellular metabolism, as SAM both hinders L-Hcys remethylation, by inhibiting 5,10-methylene-THF reductase (MTHFR) and BHMT, and allosterically activates CBS (Daubner and Matthews, 1982; Finkelstein and Martin, 1984; Finkelstein et al., 1975). Therefore, the reverse transsulfuration pathway serves the dual role of removing excess L-Hcys and producing L-Cys (Banerjee et al., 2003; Kraus et al., 1998). 


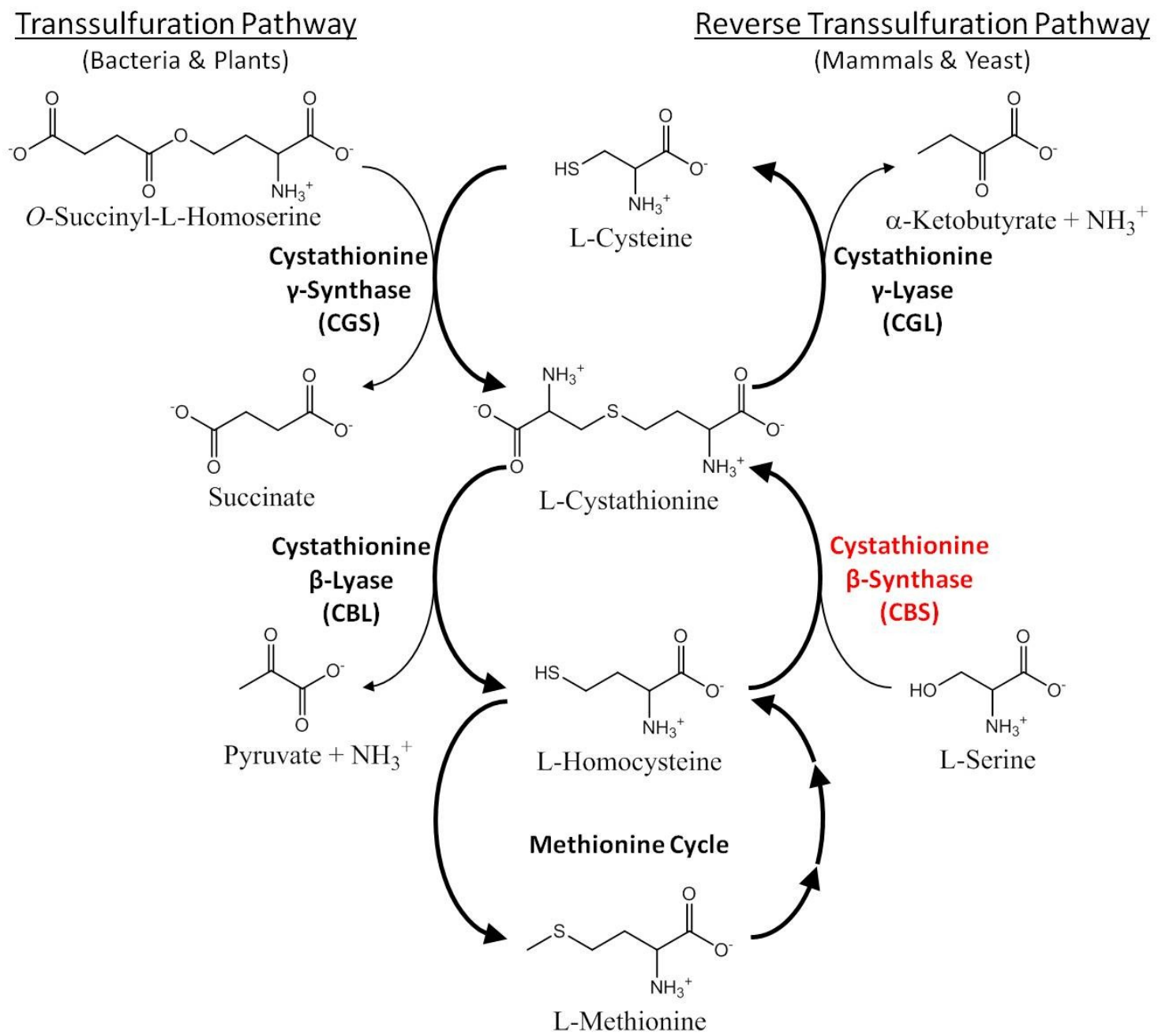

Figure 1.2: The transsulfuration pathway of bacteria and plants, the reverse transsulfuration pathway of mammals and yeast as well as their relationship with the methionine cycle (Steegborn et al., 1999). 


\subsubsection{Pyridoxal 5'-Phosphate}

Many of the enzymes involved in the metabolism of amino acids and their derivatives rely on PLP. This catalytically versatile cofactor is used in diverse reactions including decarboxylation, transamination, racemisation, and various side chain eliminations and rearrangements (Percudani and Peracchi, 2003; Clayton, 2006). Consequently, PLP-dependent enzymes are associated with 121 distinct reactions and have been assigned more than 100 Enzyme Commission (E.C.) numbers that span five of the six different enzymes classes as defined by the International Union of Biochemistry and Molecular Biology (Aitken et al., 2011; Aitken and Kirsch, 2005).

The versatility of PLP originates from its ability to function as an electrophilic catalyst, once covalently bound to an amino acid substrate via Schiff base linkage (Percudani and Peracchi, 2003). In its resting state, PLP exists in its internal aldimine form; a Schiff-base linkage with the $\varepsilon$-amino group of an active site lysine residue. Once an amino acid substrate enters the catalytic cavity, the active-site lysine residue is exchanged, via a transaldimination reaction, for the amino group of the substrate, forming the external aldimine (Hayashi, 1995; Schneider et al., 2000). The various transformations of amino acid substrates catalyzed by PLP-dependent enzymes diverge from this point (Figure 1.3). The specific reaction catalyzed by a PLP-dependent enzyme is in part determined by the orientation of the bonds at the $\mathrm{C}_{\alpha}$ atom of the substrate moiety of the external aldimine, with respect to the ring of the cofactor, which determines the $\mathrm{C}_{\alpha}$-bond to be broken (Dunathan, 1966; Christen and Metha, 2001). The pyridinium nitrogen also plays a role in reaction specificity as its electron-withdrawing properties are modulated by electrostatic and hydrogen bonding interactions with nearby residues. The 
pyridinium ring functions as an electron sink, weakening the bond of interest enabling cleavage, and subsequently stabilizes the resulting carbanion via resonance (Schneider $e t$ al., 2000; Christen and Metha, 2001).

The PLP-dependent enzymes catalyzing transformation of amino acids belong to four distinct fold types and are classified on the basis of protein sequence comparisons, available 3D structural data as well as predicted secondary structure elements (Percudani and Peracchi, 2003; Schneider et al., 2000). It has been proposed that each of the four fold types corresponds to a separate independent evolutionary lineage of PLP-dependent enzymes (Percudani and Peracchi, 2003). The largest and most thoroughly characterized of the enzyme families is Fold-type I, which includes aminotransferases, decarboxylases, and enzymes catalyzing $\alpha-, \beta$-, and $\gamma$-elimination reactions (e.g. CGL and the enzymes of the bacterial and plant transsulfuration pathways). Fold-type II enzymes primarily catalyze $\beta$-elimination and replacement reactions and often possess a regulatory domain as well as a catalytic domain (Schneider et al., 2000) (Figure 1.4). Examples of Fold-type II enzymes include $O$-acetylserine sulfhydrylase (OASS), the $\beta$-subunit of tryptophan synthase (TrpS), threonine deaminase (TD), threonine synthase (TS), and CBS. Although the overall architecture of enzymes in this fold type may vary depending on the position of regulatory domain(s), each catalytic core shares the same overall fold. The catalytic domain consists of 2 subdomains of approximately equal size, with the active site situated in the cleft formed at their interface (Schneider et al., 2000; Eliot and Kirsch, 2004). Fold types III and IV encompass relatively few members, compared to fold types I and II, and are exemplified by alanine racemase and D-Alanine aminotransferase, respectively (Percudani and Peracchi, 2003). 


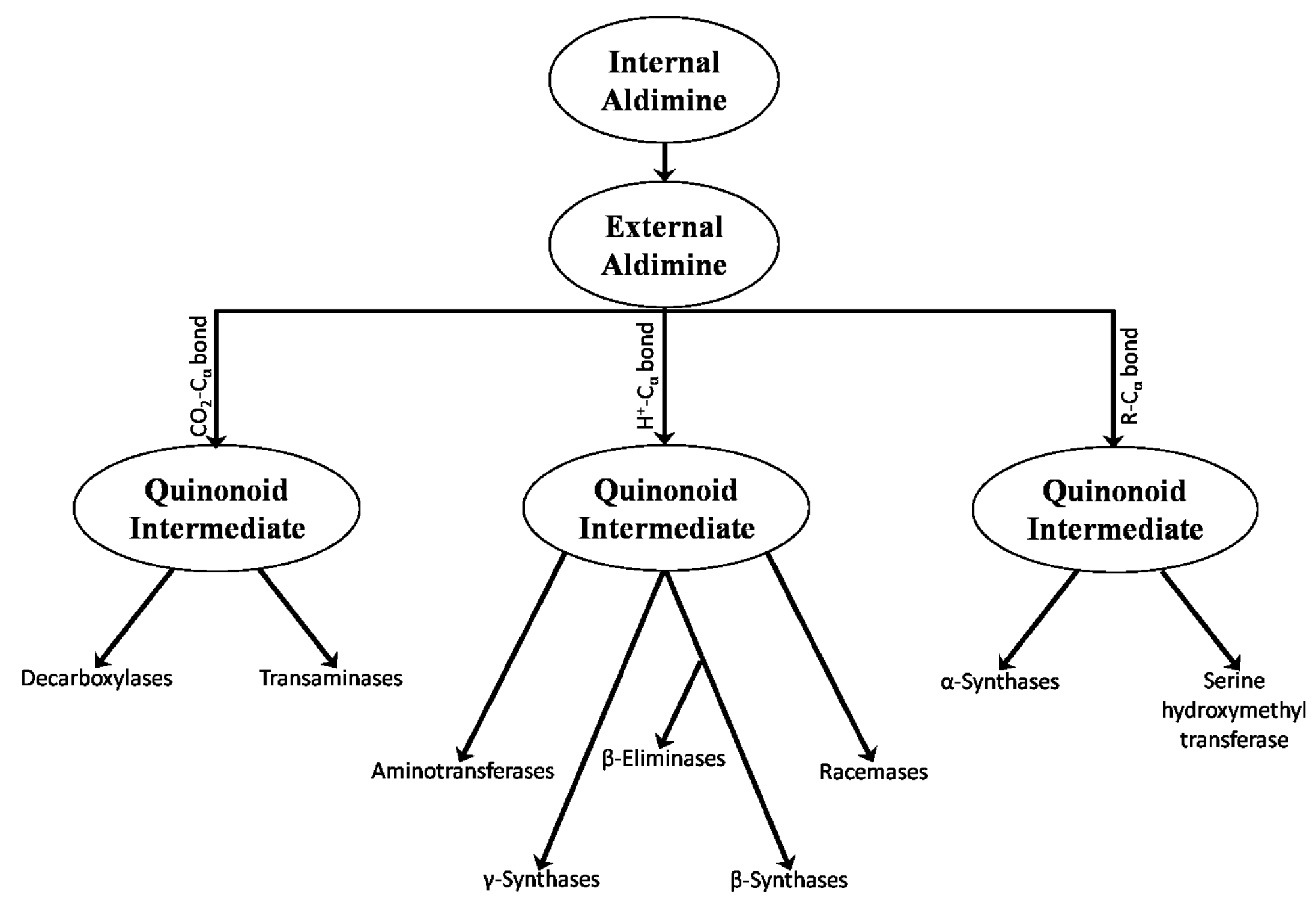

Figure 1.3: The variety of reactions catalyzed by PLP-dependent enzymes. The orientation of the substrate moiety of the external aldimine with respect to the plane of the cofactor ring determines which $\mathrm{C}_{\alpha}$-bond is to be cleaved (Dunathan, 1966; adapted from Schneider et al., 2000). 


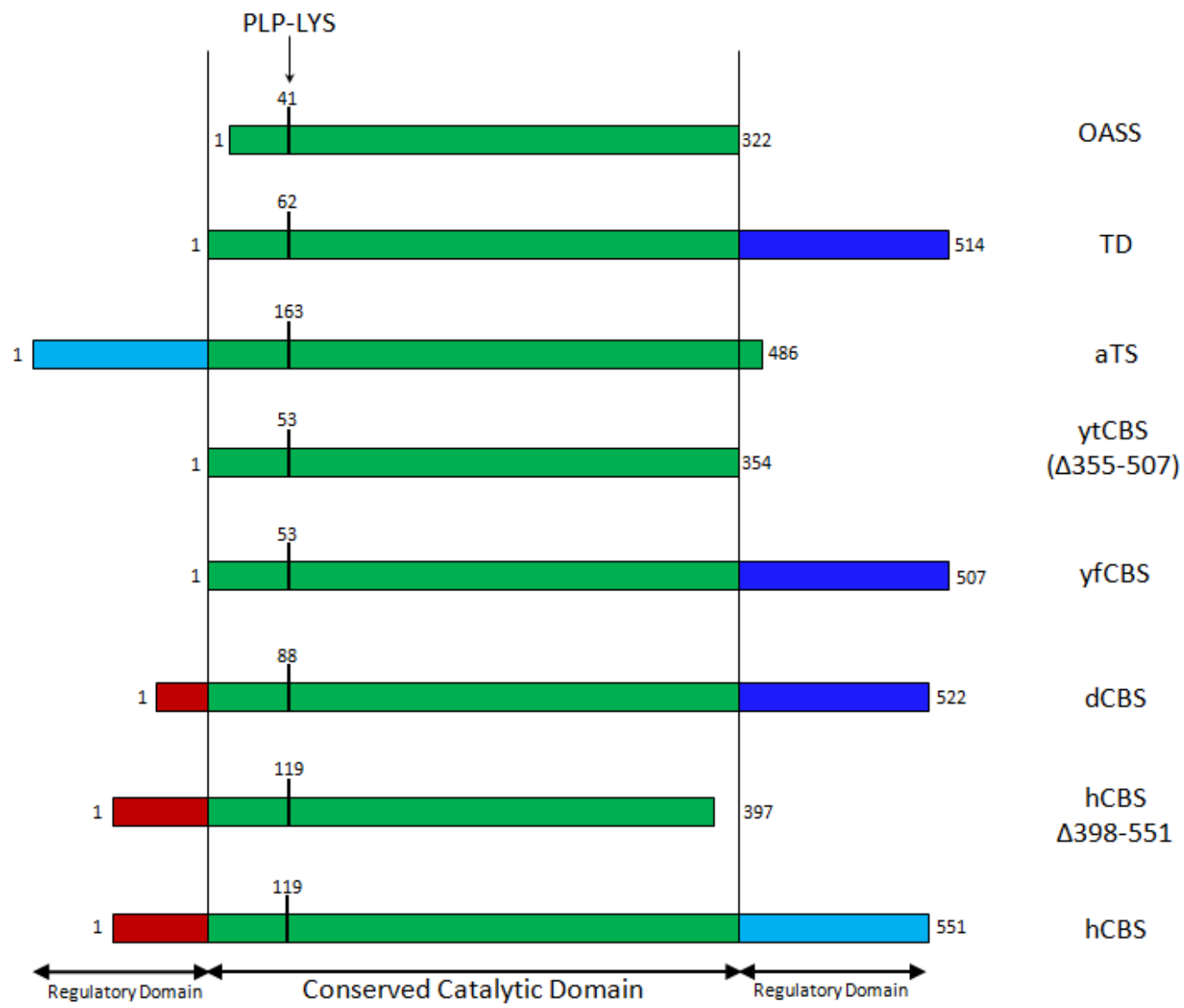

Figure 1.4: A comparison of the organization of domains among a selection of representative fold-type II PLP-dependent enzymes. Domains coloured in dark red represent the heme-binding region that is specific to CBS from higher eukaryotes, green represents the catalytic, PLP-binding region, light blue domains are those activated by Sadenosylmethionine (SAM) while dark blue domains of some CBS enzymes possess sequence and structural similarity with those activated by SAM, but have been reported to be nonresponsive to SAM (Adapted from Miles and Kraus, 2004). OASS: $O$ Acetylserine sulfhydrylase, TD: Threonine deaminase, aTS: Arabidopsis thaliana Threonine synthase (Mas-Droux et al., 2006), ytCBS: Yeast truncated Cystathionine $\beta$ synthase (residues 1-354), yfCBS: Yeast full-length Cystathionine $\beta$-synthase, dCBS: Drosphila melanogaster Cystathionine $\beta$-synthase, hCBS: Human Cystathionine $\beta$ synthase, hCBS $\triangle 398-551$ : truncated form of hCBS employed in this study. 


\subsection{Cystathionine $\beta$-synthase}

\subsubsection{Protein Architecture}

Higher eukaryotic CBS enzymes such as the human (hCBS) and Drosophila melanogaster (dCBS) homologues are unique in that they are the only PLP-dependent enzymes that also possess a heme cofactor. In contrast, CBS of lower eukaryotic forms such as yeast (yCBS) does not possess the heme-binding domain. (Kery et al., 1994; Jhee et al., 2000a; MacLean et al., 2000). The human CBS enzyme, spanning 551 residues, is a homotetramer of $63 \mathrm{kDa}$ subunits. Each subunit comprises 3 distinct domains: a Nterminal, heme-binding domain spanning $\sim 70$ residues, a central catalytic core of $\sim 340$ residues, which binds one PLP molecule in Schiff base linkage with active-site lysine (K119), and a 140 residue C-terminal, regulatory domain (Kery et al., 1999; Meier et

al., 2001). Due to the tendency of the full-length CBS enzyme to aggregate, the only crystal structure available for CBS for many years was the truncated form of hCBS, which lacks the C-terminal domain (Meier et al., 2001). Recently, the structures of the full-length Drosphila melanogaster CBS (dCBS) enzyme and a deletion variant of fulllength hCBS, lacking residues 516-525 (hCBS $\Delta 516-525)$, were reported (Koutmos et al., 2010; Oyenarte et al., 2012; Ereno-Orbea et al., 2013a). The 10-residue (516-525) segment of hCBS has been proposed to participate in higher order oligomerization of the enzyme and their removal enabled the successful crystallization of full-length hCBS (Ereno-Orbea et al., 2013a).

In higher eukaryotes, the heme-binding domain lacks secondary structure, with the exception of a short $3_{10}$ helix. Notwithstanding the lack of secondary structure, this domain is able to tightly bind the heme molecule via the axial ligands C52 and H65 
(hCBS numbering) (Ojha et al., 2000; Meier et al., 2001). Substitutions to the heme axial ligands lead to a decrease of activity to as low as $\sim 10 \%$ of the wild-type enzyme while maintaining a similar PLP content to the wild-type suggesting that this domain modulates enzyme activity (Ojha et al., 2002; Singh et al., 2009a; Smith et al., 2012). Removal of the heme-binding domain of hCBS results in a hemeless variant that retains $\sim 40 \%$ of its activity compared to the wild-type enzyme thus providing proof that heme is not vital to activity (Evande et al., 2004). The fact that lower eukaryotic CBS enzymes, such as $\mathrm{yCBS}$, do not possess a heme-binding domain and yet perform the same overall reaction further indicates that heme is not required for catalysis in hCBS (Jhee et al., 2000a; Jhee et al., 2000b).

Similar to other Fold-type II enzymes, CBS interconverts between an open, solvent-accessible active site where both substrate and product can freely enter the cavity, and a more restrictive closed conformation where the catalytic intermediates are shielded from the solvent (Aitken and Kirsch, 2005). A crystal structure of the L-Met complex of the K41A variant of the closely related OASS first established a model of the closed conformation. It revealed a "mobile loop" that undergoes a conformational change, upon substrate binding, that triggers the closing of the active site (Burkhard et al., 1999). The shift of the mobile loop was also evident in the comparison of the crystal structure of fulllength dCBS in its open and closed conformations (Koutmos et al., 2010). Once in the closed state, residues of the mobile loop form a hydrogen bonding network with the carboxylate and hydroxyl moieties of the L-Ser substrate. Substitution of residues within this hydrogen bonding network in yCBS leads to a competing $\beta$-elimination reaction, not observed for the wild-type enzyme, in which L-Ser is hydrolyzed to produce pyruvate and 
$\mathrm{NH}_{3}$. This suggests a shift in protein equilibrium towards the open conformation and increased exposure of reaction intermediates to solvent, thus demonstrating the subtle structural features that regulate catalysis and reaction specificity (Aitken and Kirsch, 2004; Aitken et al. 2011).

While analysis of the crystal structure of the OASS-K41A variant was critical in determining the active site residues involved in L-Ser binding in CBS, as it was the only ligand complex available for a fold-type II enzyme at the time, uncovering the active site residues that bind the L-Hcys substrate has proven more difficult. The OASS-K41A-LMet complex has provided very little insight into this matter as the second substrate in the OASS-catalyzed reaction is hydrogen sulfide $\left(\mathrm{H}_{2} \mathrm{~S}\right)$, which lacks the $\alpha$-carboxylate and amino groups of L-Hcys, and subsequent CBS structures have not included ligands binding at this substrate site. Lodha and colleagues (2009) probed the role of conserved polar residues in the vicinity of the proposed L-Hcys binding site and determined that although these residues play a significant role in sustaining proper active-site conformation, their side chains are not direct determinants of L-Hcys binding (Lodha et al., 2009).

The recent report of the full-length hCBS $(\Delta 516-525)$ structure, which exhibits similar basal enzyme activity to native hCBS and is activated by SAM, provides the structural basis for studies probing the mechanism of hCBS regulation (Oyenarte et al., 2012; Ereno-Orbea et al., 2013a). The pairing of the catalytic core domains of the $\Delta 516$ 525 variant is similar to that of $\mathrm{dCBS}$ but the relative positioning of the $\mathrm{C}$-terminal domain of the two CBS enzymes is distinct (Figure 1.5). The regulatory domain of dCBS interacts with the catalytic core only through the linker. As a result, substrates have 
unrestricted entry to the dCBS active site (Koutmos et al., 2010; Ereno-Orbea et al., 2013b). Conversely, the SAM-binding domain of the hCBS ( $\Delta 516-525)$ dimer is at the head of the active site of the complementary subunit, hindering substrate access to the cavity (Ereno-Orbea et al., 2013a; Ereno-Orbea et al., 2013b) (Figure 1.5). As a result, the hCBS $(\Delta 516-525)$ dimer forms a basket-shaped symmetrical dimer where the catalytic core of each subunit interacts with both the catalytic and regulatory domain of the neighboring subunit (Figure 1.5). The auto-inhibitory feature of the SAM-binding domain is due to the presence of four loops at the mouth of the active site. SAMbinding is believed to rotate the position of the CBS motifs causing the relaxation of the four loops and provide easier substrate entry into the active site (Ereno-Orbea et al., 2013a). Insights into the crystal structure have led to a proposal wherein hCBS tetramerization occurs as an assembly of 2 dimers stabilized by a loop containing residues 513-519 and the absence of residues 516-525 in hCBS may have allowed for this particular structure to be crystallized (Ereno-Orbea et al., 2013a). 

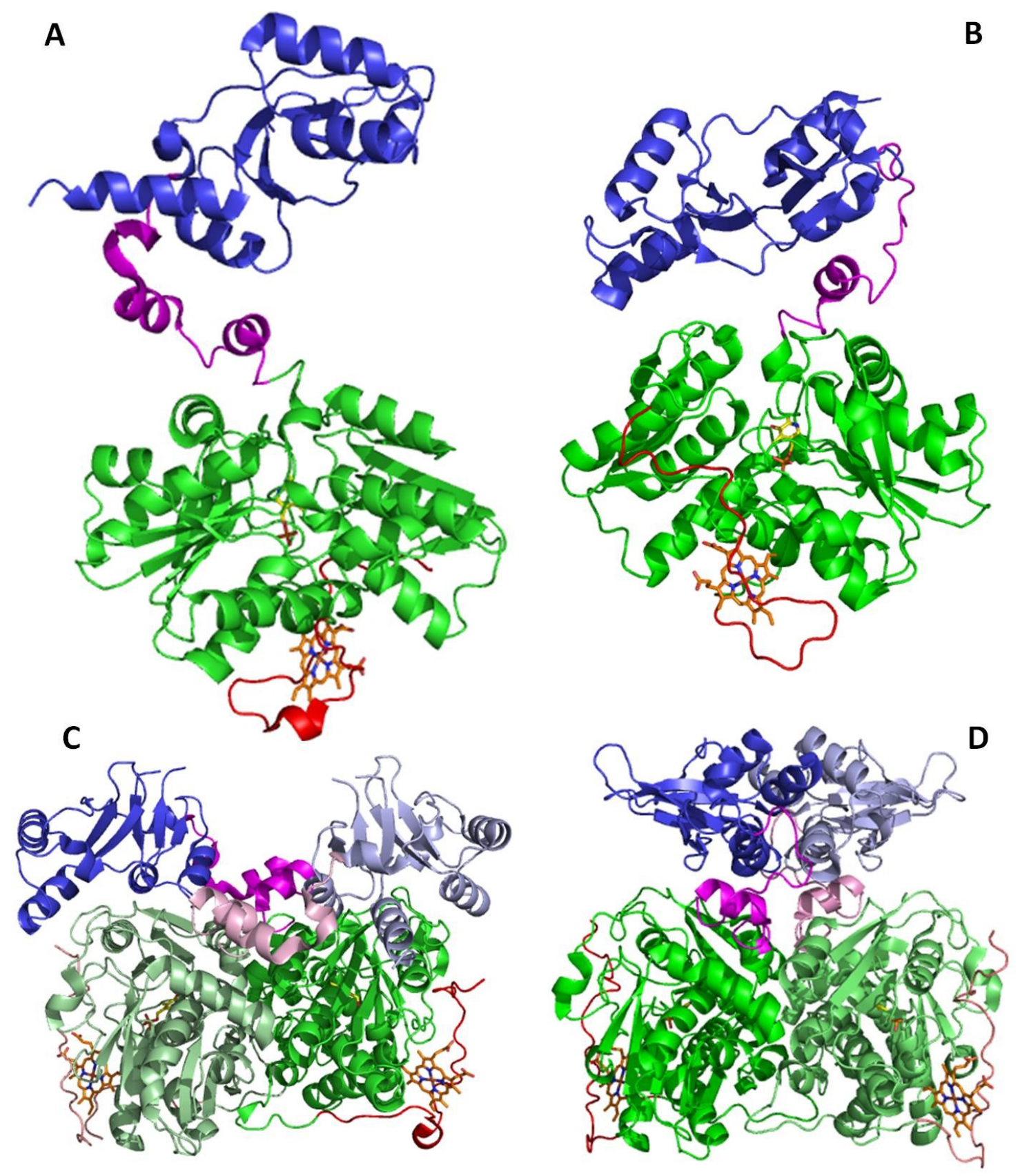

Figure 1.5: Structural differences in the positioning of the regulatory domain in the hCBS $(\Delta 516-525)$ and dCBS protein structures. (A and B): $h C B S(\Delta 516-525)(\mathrm{A})$ and $\mathrm{dCBS}(\mathrm{B})$ monomers showing the heme-binding domain (red), catalytic core (green), $\mathrm{C}$ terminal domain (blue), and the linker (magenta) that connects the catalytic and $\mathrm{C}$ terminal domains. (C and D): the dimeric structures of hCBS (C) and dCBS (D) demonstrating the placement of one subunit (bright colours) relative to the complementary subunit (pale colours) (Koutmos et al., 2010; Ereno-Orbea et al., 2013a). Heme: Orange, PLP: Yellow 


\subsubsection{Reaction Mechanism}

CBS catalyzes the condensation of L-Ser and L-Hcys to form the transsulfuration intermediate L-Cth. The presence of heme within hCBS masks the spectroscopic detection of PLP intermediates, and so the hemeless yCBS homologue has been used in studies investigating the reaction mechanism and the effect of site-directed substitutions of active-site residues. Yeast CBS is an suitable model system for these studies as it catalyzes the same overall reaction and shares $47 \%$ amino acid identity with the catalytic core of hCBS (Jhee et al., 2000a; Jhee et al., 2000b).

The $\alpha$-amino group of the L-Ser substrate nucleophilically attacks the electronpoor carbon ( $\mathrm{C}^{\prime}$ ') atom of the internal aldimine leading to the formation of the first transient germinal diamine, followed by formation of the external aldimine (E-Ser) via the displacement of $\varepsilon$-amino group of the active-site lysine (hCBS-K119, yCBS-K53) (Figure 1.6). Abstraction of the $\mathrm{C} \alpha$ proton of the L-Ser substrate by the $\varepsilon$-amino of the released lysine residue leads to the displacement of the $\beta$-hydroxyl group of the substrate and the formation of the aminoacrylate intermediate (Figure 1.6). The free thiol group of L-Hcys then nucleophilically attacks the aminoacrylate intermediate leading to the formation of the external aldimine of L-Cth (E-Cth) (Figure 1.6). The rate-limiting step of this reaction is the release of the L-Cth product, via nucleophilic attack on the electrondeficient C4' atom by the catalytic lysine, regenerating the internal aldimine (Figure 1.6) (Jhee et al., 2001). Both yCBS and hCBS have demonstrated the ability to catalyze the reverse-physiological hydrolysis of L-Cth, producing L-Ser and L-Hcys (Jhee et al., 2001; Belew et al., 2009). 


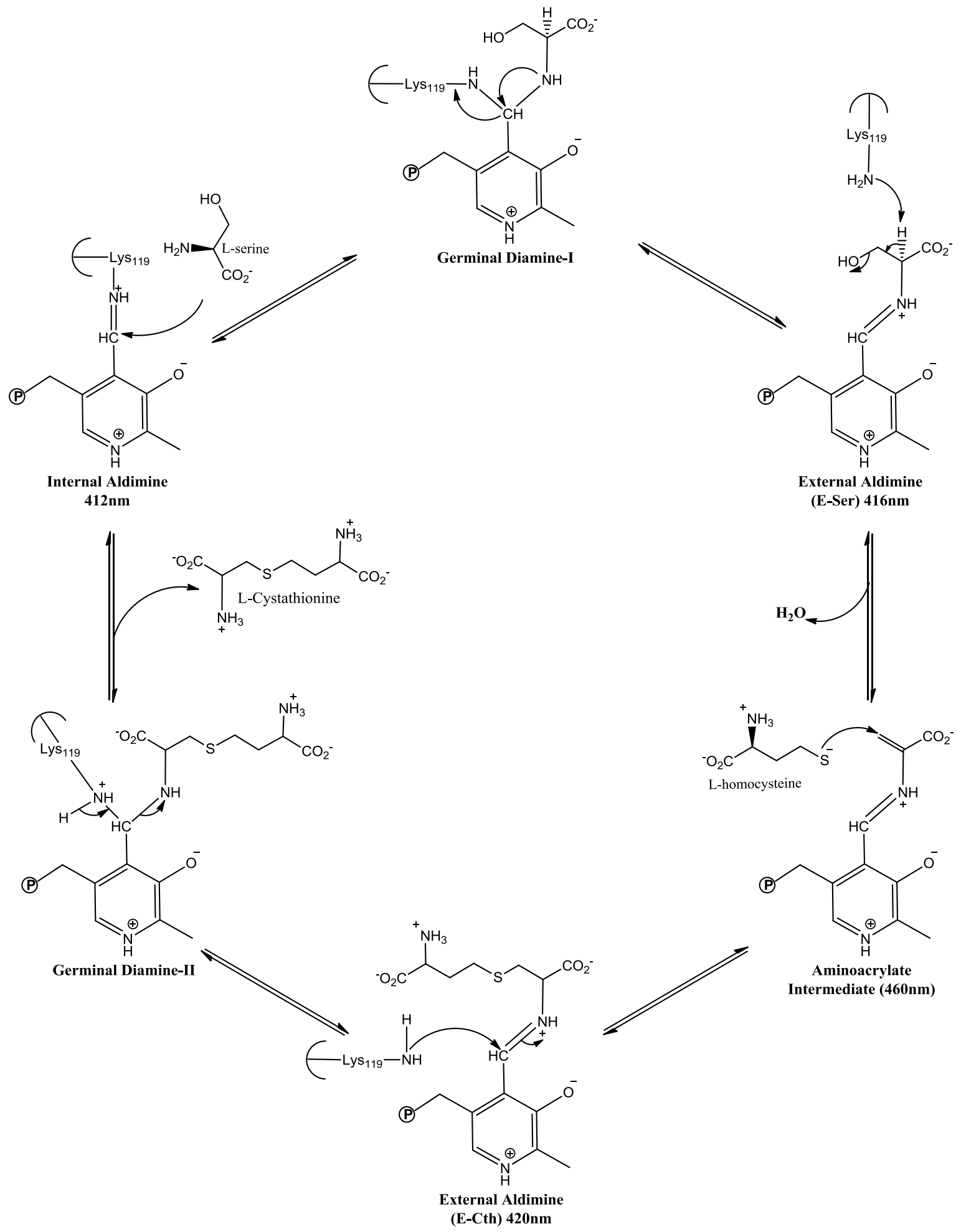

Figure 1.6: Reaction mechanism of CBS, showing the PLP-bound intermediates as well as their associated absorption maxima, as observed in yCBS (Jhee et al., 2001). E-Ser: external aldimine of L-Ser, E-Cth: external aldimine of L-Cth. 
Both yCBS and hCBS are susceptible to substrate inhibition (Jhee et al., 2000a; Belew et al., 2009). At physiological concentrations (L-Ser: $\sim 560 \mu \mathrm{M}$; L-Hcys: $\sim 10 \mu \mathrm{M}$ ), L-Ser out-competes L-Hcys for access to the free enzyme (Singh et al., 2009b; Jhee et al., 2000a). At elevated L-Hcys concentration, it occupies the L-Ser binding site, effectively reducing the pool of available free enzyme to which L-Ser binds (Jhee et al., 2000a). A second mode of inhibition arises from the premature binding of L-Hcys to E-Ser before the release of $\mathrm{H}_{2} \mathrm{O}$ leading to the formation of an inactive enzyme-serine-homocysteine (E-Ser-Hcys) complex (Jhee et al., 2000a).

There are many interactions between the PLP cofactor and the active site residues of hCBS that regulate the orientation and electronic properties of the cofactor as well as define the active site architecture and dynamics, which collectively regulate substrate and reaction specificity as well as the rate of catalysis. For example, interactions with the pyridinium nitrogen $(\mathrm{N} 1)$, hydroxyl group $\left(\mathrm{O}^{\prime}\right)$ and the phosphate moiety of the cofactor can manipulate its electronic properties (Lodha et al., 2010). Similar to other Fold-type II PLP-dependent enzymes, hCBS-S349 (yCBS-S289) is essential for modulating the electron withdrawing properties and orientation of the pyridine ring of PLP via a hydrogen bond with the N1 atom of pyridinium ring (Eliot and Kirsch, 2004; Quazi and Aitken, 2009). Residue N149 of hCBS (yCBS-N84) forms a hydrogen bond with the hydroxyl moiety (O3') of PLP and, like hCBS-S349, is believed to maintain the orientation of the pyridine ring while playing a crucial role in preserving PLP in its active ketoenamine state (Lodha et al., 2010; Weeks et al., 2009). Residue G305 (hCBS numbering) may maintain the orientation of the pyridine ring through hydrophobic interactions between the $\mathrm{C}_{\alpha}$ of G305 and the pi system of the cofactor's aromatic ring 
(Meier et al., 2003). The mobile loop (residues T146-S147-G148-N149-T150 of hCBS, conserved as T81-S82-G83-N84-T85 in yCBS) plays an important role in the coordination of the $\alpha$-carboxyl group of the L-Ser substrate while the backbone of G307, positioned at the mouth of the active site, is thought to play a role in L-Hcys binding (Aitken and Kirsch, 2004; Meier et al., 2003). Residues S147, K177, E304, K384, E176 and T383 of hCBS are part of a hydrogen bonding network that bridges the mobile loop and residues of the C-terminus of the catalytic domain (D376, R379, S380, Y381) (Lodha et al., 2009; Lodha et al., 2010). A glycine-rich loop (hCBS-G256-T257-G258-G259T260) wraps around the phosphate moiety with the two threonine residues (T257 and T260) providing hydrogen bonding linkage to PLP (Banerjee et al., 2003; Yadav et al., 2012). Understanding the correlation between the electrostatic and hydrogen bonding properties of residues within the active site and enzymatic function is critical to explaining how modifications to these interactions, caused by homocystinuria-associated mutations, may alter the ability of the enzyme to perform its canonical role and/or its secondary role as a $\mathrm{H}_{2} \mathrm{~S}$ producer.

\subsubsection{Role of CBS in Hydrogen Sulfide Production}

Recent studies have indicated that $\mathrm{H}_{2} \mathrm{~S}$ mediates a wide range of physiological responses such as muscle relaxation, the induction of the intracellular signal transducer cyclic adenosine monophosphate (cAMP) and enhancing the flow of cations into the cell by way of NMDA receptors (Abe and Kimura, 1996; Kimura, 2013; Kimura, 2000). The concentration of $\mathrm{H}_{2} \mathrm{~S}$ in vivo is in the tens to hundreds micromolar range (Singh and Banerjee, 2011; Evans, 1967). In mammalian tissues, $\mathrm{H}_{2} \mathrm{~S}$ is chiefly produced by CBS 
and CGL with the majority of $\mathrm{H}_{2} \mathrm{~S}$ production in the brain attributed to CBS, while in other tissues such as the small intestine and the thoracic aorta $\mathrm{H}_{2} \mathrm{~S}$ is produced by CGL (Hosoki et al., 1997). $\mathrm{H}_{2} \mathrm{~S}$ increases GSH production in reactive astrocytes, suggesting a role in the protection of neurons from oxidative stress (Kimura and Kimura, 2004; Kimura, 2013).

Three distinct $\beta$-replacement reactions resulting in the production of $\mathrm{H}_{2} \mathrm{~S}$ have been reported for CBS (Figure 1.7): the condensation of L-Cys (replacing L-Ser) with LHcys, leading to the production of L-Cth and $\mathrm{H}_{2} \mathrm{~S}$ [2], the condensation of two molecules of L-Hcys to form L-lanthionine and $\mathrm{H}_{2} \mathrm{~S}$ [3] and exchange of the sulfhydryl moiety of LCys with a hydroxyl group, from water, to form L-Ser and $\mathrm{H}_{2} \mathrm{~S}$ [4] (Yadav and Banerjee, 2012). Reaction [2] (the condensation of L-Ser and L-Hcys) is the principal in vivo $\mathrm{H}_{2} \mathrm{~S}$ generator, encompassing $\sim 96 \%$ of the total amount of $\mathrm{H}_{2} \mathrm{~S}$ produced in the brain (Singh et al., 2009b). The generation of $\mathrm{H}_{2} \mathrm{~S}$ is generally believed to depend on the availability of L-Cys rather than L-Hcys, since L-Cys is capable of functioning as both the first and second substrates in a CBS-catalyzed reaction while L-Hcys can only be the second substrate, and because $\mathrm{H}_{2} \mathrm{~S}$ production by CBS is relatively insensitive to increasing LHcys concentration (Singh and Banerjee, 2011; Singh et al., 2009b). This is further supported by the $\sim 1-2$-fold order-of-magnitude difference between the expected intracellular concentration of L-Cys $(\sim 200 \mu \mathrm{M})$ and the $K_{m}$ values of L-Cys with respect to the two substrate binding sites $(6.8 \mathrm{mM}$ and $27 \mathrm{mM}$, respectively) (Singh and Banerjee, 2011; Özkan et al., 2002). Consequently, elucidating the roles of active site residues of CBS may provide insight into its diverse physiological roles. 
Figure 1.7: The four known $\beta$-replacement reactions catalyzed by CBS. Reaction 1 represents the canonical reaction while reactions 2-4 characterize the possible $\beta$-replacement reactions that yield $\mathrm{H}_{2} \mathrm{~S}$ (Yadav and Banerjee, 2012) 


\subsubsection{Proposed Roles of Heme in hCBS}

The role of the heme cofactor has yet to been determined and several hypotheses have been proposed over the past two decades (Taoka et al., 1998; Janosik et al., 2001b; Majtan et al., 2011). Majtan and colleagues (2008) demonstrated the production of stable and active hCBS in heme-deficient strains of Saccharomyces cerevisiae and Escherichia coli using chemical chaperones, porphyrin rings lacking a metallic core and exogenous heme. In a subsequent study, Majtan et al. (2011) described the purification of a fulllength human enzyme with a cobalt-centred protoporphyrin IX ring substituted for heme. This approach yielded an enzyme with no significant change in specific activity, quaternary structure, and responsiveness to SAM and PLP saturation, compared to native hCBS (Majtan et al., 2011). The authors concluded that heme cofactor serves a structural role within the enzyme (Majtan et al., 2011). However, a consensus as to the role of heme has not been reached, as other researchers hypothesized the cofactor functions as a redox sensor (Taoka et al., 1998).

In its ferric $\left(\mathrm{Fe}^{3+}\right)$ state, the hCBS heme is relatively inert to ligand exchange with exogenous compounds, while in its ferrous $\left(\mathrm{Fe}^{2+}\right)$ state, the iron atom is able to bind carbon monoxide (CO) and nitric oxide (NO) (Taoka and Banerjee, 2001; Banerjee and Zou, 2005). Binding of these molecules displaces C52 and concommitantly induces a tautomeric shift of PLP from its ketoenamine (active) form to its enolimine (inactive) state (Weeks et al., 2009). The shift is believed to be induced by the removal of a salt bridge between C52 and R266, located at the C-terminal end of $\alpha$-helix 8 (Figures $1.8 \&$ 1.9). The resulting deprotonation of the nitrogen atom strengthens the imine bond and decreases the susceptibility of the adjacent C4' atom to a nucleophilic attack by L-Ser 
(Figure 1.8). As a result of the tautomeric shift, the ketoenamine-stabilizing hydrogen bond between the O3' atom of PLP ring and active-site residue Asn149 is lost (Weeks et al., 2009) (Figure 1.9).

Residue N84 of yCBS plays a role in reaction specificity as well as PLP orientation within the active site (Lodha et al., 2010). Substitution of N84 in yCBS, the residue corresponding to hCBS-N149, is believed to cause a shift in the orientation of PLP within the active site, evident by the $\sim 230$-fold reduction in catalytic efficiency in addition to the development of marginal $\beta$-elimination activity (Lodha et al., 2010). Residues located at the active-site end of $\alpha$-helix 8 are involved in the stabilization of PLP via hydrogen bonding interactions with its phosphate moiety. Alteration of these residues resulted in the destabilization of PLP, while increasing the susceptibility to enzyme inactivation when bound to ferrous heme (Yadav et al., 2012). This has led to the suggestion of bi-directional communication between PLP and heme through $\alpha$-helix 8 (Yadav et al., 2012). This brings credence to heme's proposed role as a redox regulator as the oxidation state of iron can be relayed to the PLP site via $\alpha$-helix 8 , with resulting modulation of enzymatic function. 


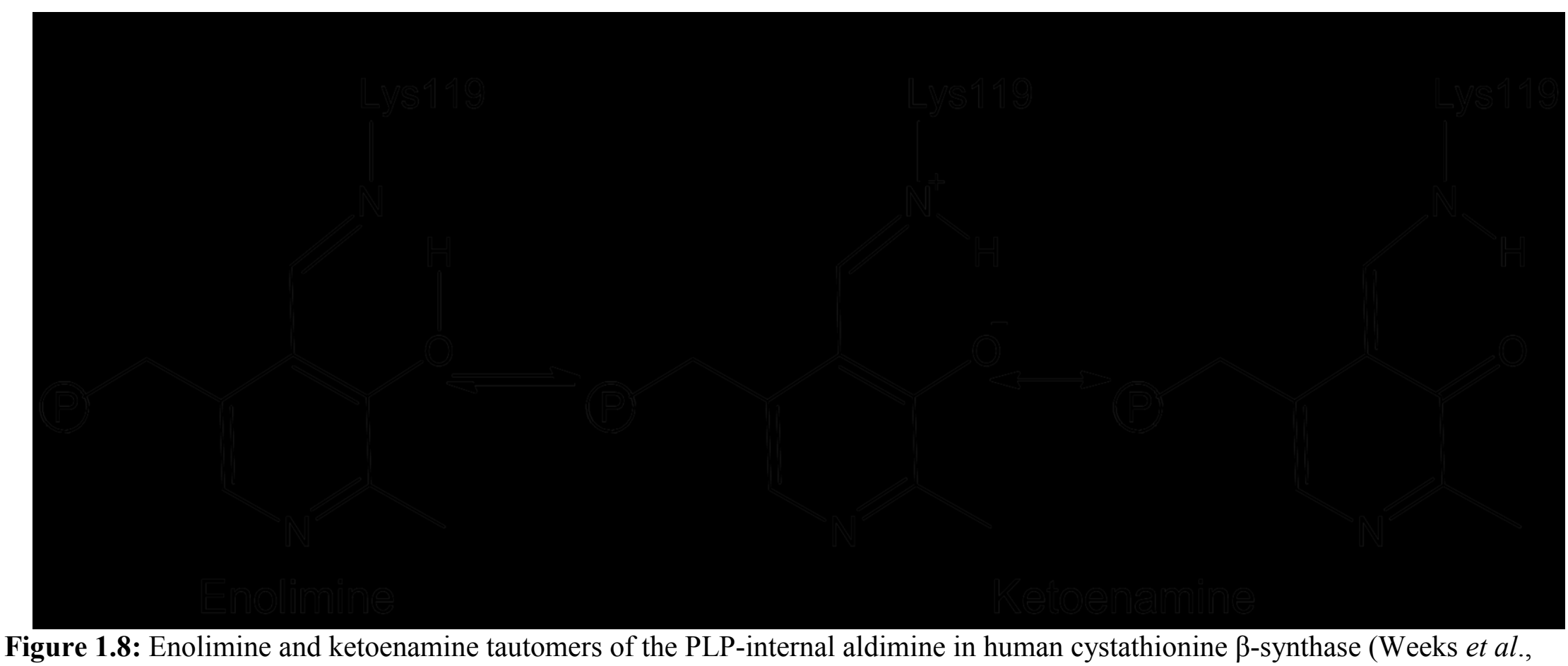
2009) 
$\mathrm{OH}$

$\mathrm{Thr}_{257}$

Figure 1.9: Proposed mechanism for the heme-CO linked inactivation of hCBS (Weeks et al., 2009). Targeted residues of this study are highlighted in red. (A) Within the hCBS active site, CO replaces the thiol of C52 as a heme ligand resulting in the loss of the ketoenamine-stabilizing hydrogen bond between the O3' atom of PLP ring and N149. (B) Proposed contacts of corresponding residues in yCBS, which lacks the heme-binding region. 


\subsubsection{SAM Regulation in $C B S$}

The C-terminal domain of hCBS contains a tandem repeat of a characteristic $\beta-\alpha-$ $\beta-\beta-\alpha$ fold, known as a "CBS" domain due to its initial discovery in hCBS (Bateman, 1997). These domains are found in other enzymes, including inosine monophosphate dehydrogenases, membrane channels and transporters, such as members of the $\mathrm{ABC}$ transporter family that transport glycine, betaine and L-proline (Bateman, 1997). Each hCBS monomer contains two such domains, designated CBS1 and CBS2, which are proposed to bind the allosteric regulator SAM, although the mechanism of activation remains to be elucidated (Finkelstein et al., 1975; Ereno-Orbea et al., 2013a). In the absence of SAM, hCBS activity is limited to a "basal" level by the autoinhibitory action of the C-terminal domain (Janosik et al., 2001a; Scott et al., 2004). Upon either SAM binding or removal of the C-terminal regulatory domain hCBS activity is increased $~ 2-4$ fold (Kery et al., 1998; Evande et al., 2002). While not activated by SAM, removal of the C-terminal domain of yCBS results in the same increase in activity observed for hCBS (Jhee et al., 2000a). In contrast, the opposite effect occurs in dCBS when the C-terminal domain is removed (Koutmos et al., 2010). This suggests that there is a difference in the arrangement of CBS domains such that it leads to a higher basal activity in dCBS while limiting basal activity in hCBS (Koutmos et al., 2010; Ereno-Orbea et al., 2013a).

Recent work has shown that there are two potential SAM-binding sites (S1 and S2) within the C-terminal domain of hCBS (Pey et al., 2013) (Figure 1.10). Both sites are located in separate cavities between CBS1 and CBS2 and are structurally similar to sites reported to bind adenosyl ligands in other proteins with tandem CBS domains (Pey et al., 2013; Ereno-Orbea et al., 2013a). Both sites possess a hydrophobic pocket of residues 
that can accommodate an adenine ring as well as conserved asparagine and threonine residues for interactions with the ribose moiety (Ereno-Orbea et al., 2013a). Isothermal titration calorimetry (ITC) data has demonstrated that both proposed binding sites of hCBS are functional and that S2 functions as a high-affinity site, responsible for increasing the stability of the enzyme, while subsequent binding of SAM to the loweraffinity S1 site results in enzyme activation (Pey et al., 2013). A mechanism of hCBS activation by SAM has recently been proposed in which SAM-binding to S2 causes both CBS motifs to rotate away from the catalytic core, thus exposing S1 for further SAMbinding (Pey et al., 2013; Ereno-Orbea et al., 2013a).

\subsubsection{Homocystinuria-associated Mutations of CBS}

Missense mutations constitute $\sim 87 \%$ of all CBS mutations associated with homocystinuria and are not restricted to a particular region of the enzyme (Majtan et al., 2010). This is exemplified by the location of the five most common missense mutations: I278T (subunit interface), T191M (catalytic core), G307S (catalytic core), R336C (subunit interface) and D444N (SAM-binding domain) (Meier et al., 2003; Yamanishi et al, 2006). Some CBS missense mutations associated with homocystinuria do not specifically target catalytic residues and result in the production of misfolded proteins, impairing function of protein levels (Majtan et al., 2010). A thorough understanding of the structure-function relationships underlying the homocystinuria-associated mutations on the enzyme will provide insight for the enhancement of therapeutic treatments. 


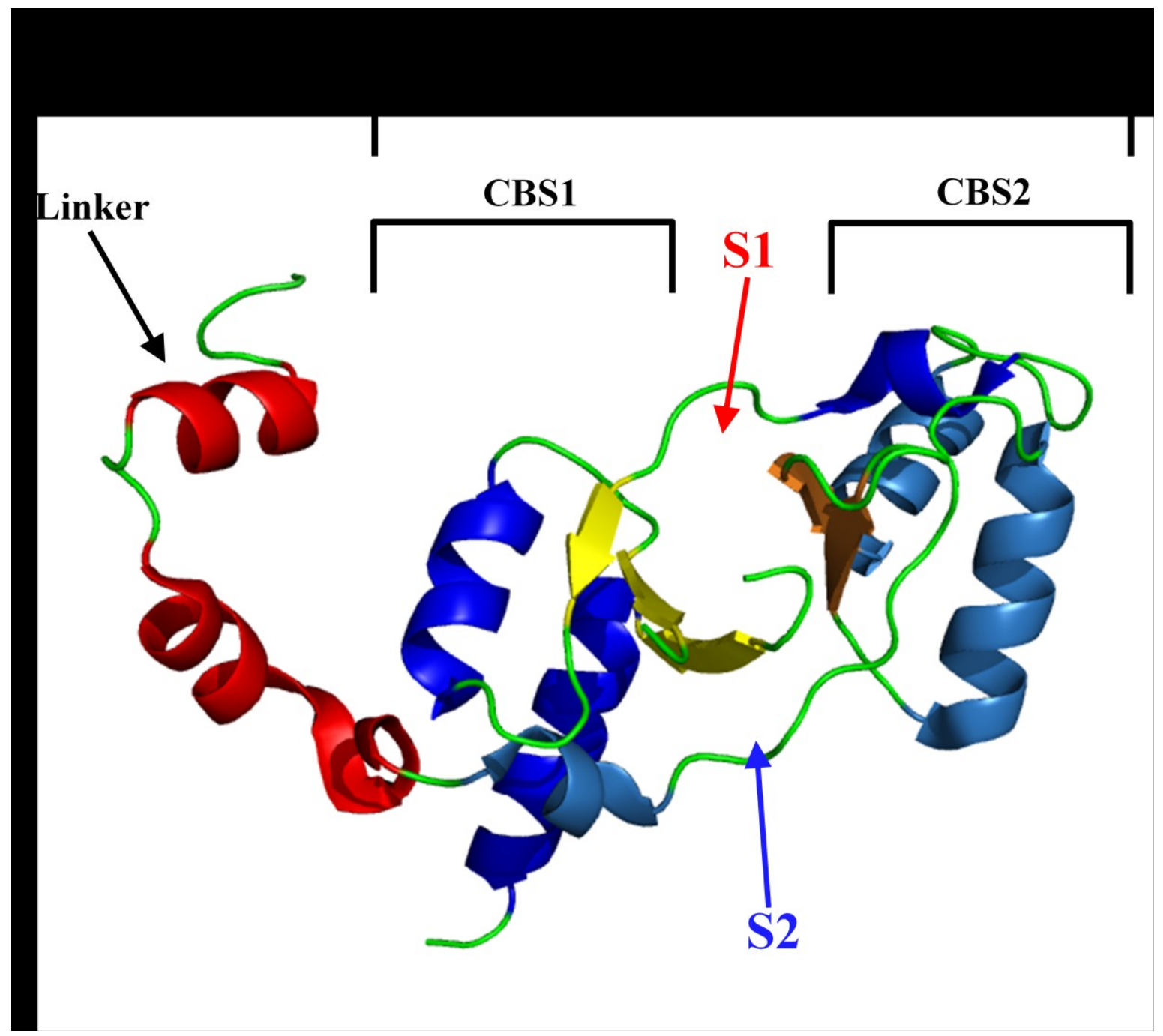

Figure 1.10: A $3 \mathrm{D}$ model of the hCBS $\Delta 516-525$ variant crystal structure showing both CBS domains (CBS1 and CBS2) and the location of the proposed binding sites of SAM (S1 and S2) (Ereno-Orbea et al., 2013a). 


\subsection{Focus of Study and Objectives}

Treatment of hereditary metabolic disorders, such as homocystinuria, is complicated by the need to consider the specific allele(s) an individual possesses when developing a treatment regime. For example, $\mathrm{B}_{6}$ supplementation is only effective for approximately half of reported disease-associated alleles. This necessitates development of an understanding the structure-function relationships that underlie each distinct disease-associated allele and how these are manifested in vivo, as well as clinically. This study explores these structure-function relationships in the active site of the enzyme cystathionine $\beta$-synthase. The objectives of this study are:

1. Investigation of residues D319, S323 and Y324 in yCBS, located at the junction of the catalytic domain and the linker segment that connects the catalytic and regulatory domains. These residues are proposed to position active site residue yCBS-N84 (hCBS-N149), which regulates reaction specificity and PLP positioning (Lodha et al., 2010). Site-directed mutagenesis was employed to remove only the polar group of each residue (D319A, S323A and Y324F) in order to probe its role within the active site hydrogen bonding network.

2. Exploration of homocystinuria-associated mutations in the hCBS active site. These included residues of the mobile loop (G148), bridging the active site cleft (E176, K384), interacting with the phosphate moiety (T257, G259) or pyridine ring (G305, S349) of the cofactor or with a proposed role in L-Hcys binding (G307). A series of 11 homocystinuria-associated variants (G148E/R, E176K, T257M, G259D/M, G305R, G307S, S349N and K384E/N) were created using a 
hCBS construct lacking residues 398-551 (hCBS CAT_L397) allowing for direct comparison with the corresponding hCBS structure (Meier et al., 2001). 


\section{METHODS}

\subsection{Materials}

Isopropyl- $\beta$-D-thiogalactopyranoside (IPTG) was obtained from BioShop. Lcystathionine, $\beta$-NADH, L-Ser and D,L-homocysteine (D,L-Hcys) were purchased from Sigma. Dithiothreitol (DTT), ampicillin, 5,5'-dithio-bis-(2-nitrobenzoic acid) (DTNB) and imidazole were acquired from Fischer Scientific. Nickel-nitriloacetic acid (Ni-NTA) resin and L-Lactate dehydrogenase (LDH) were purchased from Qiagen and Roche, respectively. The Escherichia coli cystathionine $\beta$-lyase (eCBL) coupling enzyme was expressed and purified as described by Aitken and Kirsch (2004).

\subsection{Construction of Site-Directed Mutants}

Oligonucleotide primers were designed for the introduction of the following sitedirected mutations into the yCBS full-length sequence (yfCBS): D319A, S323A and Y324F and the truncated hCBS (htCBS) variant hCBS_CAT_L397 (residues 1-397): G148E/R, E176K, T257M, G259D/M, G305R, G307S, S349N and K384E/N (Table 2.1). The S323A and Y324F yfCBS constructs were prepared by Allison Jaworski. Overlapextension polymerase chain reaction (OE-PCR), using the pSECseq0 and pSECseq7r flanking primers in addition to the mutagenic primers (Table 2.1), was utilized to construct the site-directed mutants (Higuchi et al., 1988). The re-assembled amplicons were inserted between the NdeI and HindIII (hCBS) or NdeI and PstI (yCBS) restriction sites of the pTrc3 expression vector, which encodes a N-terminal 6-His affinity tag (Farsi et al., 2009). The yfCBS and htCBS expression plasmids were transformed, via the heat shock method, with E. coli ER1821 cells (Froger and Hall, 2007). Nucleic acid sequencing was used to verify the presence of the site-directed mutations in each vector. 
Table 2.1: Primers used in the construction of the yfCBS and htCBS site-directed mutants.

\begin{tabular}{|c|c|}
\hline $\begin{array}{c}\text { yfCBS } \\
\text { Variant }\end{array}$ & Mutagenic Primer Sequence \\
\hline D319A* & $5^{\prime}$-ccatattcccaGCGtccatcaggtcg \\
\hline S323A* & 5'-gattccatcaggGCGtacctaacc \\
\hline Y324F* & $5^{\prime}$-gattccatcaggtcgTTCctaaccaa \\
\hline $\begin{array}{c}\text { htCBS } \\
\text { Variant }\end{array}$ & Mutagenic Primer Sequence \\
\hline G148E* & 5'-cgagccgacatccGAAaacaccgggatcg \\
\hline G148R* & 5' -cgagccgacatccCGTaacaccgggatcg $^{\prime}$ \\
\hline E176K* & 5'-ccagagaagatgagctccAAAaaggtggacgtgc \\
\hline T257M* & 5'-ggcttcagtgggcATGggcggcacc \\
\hline G259D* & $5^{\prime}$-ggcacgggcGACaccatcacg \\
\hline G259M* & $5^{\prime}$-ggcacgggcATGaccatcacg \\
\hline G305R* & 5' -cgaggtggaaCGTatcggctacg \\
\hline G307S* & $5^{\prime}$-ggaagggatcTCTtacgacttcatcc \\
\hline S349N* & $5^{\prime}$-gctgtgcggtggcAACgctggcagc \\
\hline K384E* & $5^{\prime}$-ggaactacatgaccGAAttcctgagcg \\
\hline K384N* & $5^{\prime}$-ggaactacatgaccAActtcctgagcg \\
\hline pSECseq0* & 5' -ggcgtcaggcagccatcggaagctg \\
\hline pSECseq7r* & $5^{\prime}-$ gcccgccaccctccgggcegttgcttcgc \\
\hline
\end{tabular}

*The second mutagenic primer is the reverse complement of the primers listed above 


\subsection{Expression and Purification of yfCBS Mutants}

All yfCBS constructs was expressed and purified using the same procedure. Three 2.8-L Fernbach flasks, each containing $1 \mathrm{~L}$ of CBS growth media $(50 \mathrm{mM}$ potassium phosphate, 50X V.B Salt, $7.2 \mathrm{~g}$ Tryptone, $14.4 \mathrm{~g}$ Yeast extract, glycerol) and $100 \mu \mathrm{g} / \mathrm{mL}$ ampicillin, were inoculated with an overnight culture (1:10 ratio) of the E. coli strain ER1821 containing the yfCBS construct. The cultures were grown at $30^{\circ} \mathrm{C}$ until the $\mathrm{OD}_{600}$ reached 0.5 before inducing with $0.15 \mathrm{mM}$ IPTG and further incubation for 16 hours. The cells were harvested via centrifugation at $5000 \mathrm{RPM}$ at $4^{\circ} \mathrm{C}$ for 10 minutes. The resulting pellets were resuspended in $0.85 \% \mathrm{NaCl}$ and centrifuged at $5000 \mathrm{RPM}$ for 15 minutes before storage at $-80^{\circ} \mathrm{C}$.

The stored cells were resuspended in buffer A (50 mM potassium phosphate, $\mathrm{pH}$ 7.8, $20 \mu \mathrm{M}$ PLP and $10 \mathrm{mM}$ imidazole) and lysozyme was added, to $1 \mathrm{mg} / \mathrm{mL}$, with subsequent incubation for 20 minutes at room temperature. Following sonication $(8 \times 30$ seconds at $50 \%$ duty cycle) the cellular debris was removed by centrifugation at 15000 $\mathrm{RPM}$ at $4^{\circ} \mathrm{C}$ for 60 minutes. The supernatant was loaded onto a $1.0 \times 10 \mathrm{~cm}$ column of NiNTA resin, equilibrated with 20 column volumes of buffer A. The affinity-tagged enzyme was eluted with a $200 \mathrm{~mL}$ linear gradient of $10-200 \mathrm{mM}$ imidazole in buffer A. The fractions collected were assessed for protein purity via SDS-PAGE and fractions containing $>95 \%$ pure target enzyme, as assessed by visual inspection, were pooled, concentrated and dialyzed against storage buffer $(50 \mathrm{mM}$ potassium phosphate, $1 \mathrm{mM}$ EDTA, pH 7.8, $1 \mathrm{mM}$ DDT \& $20 \mu \mathrm{M}$ PLP). Glycerol was added to $20 \%$ (v/v) before storage at $-80^{\circ} \mathrm{C}$. The protein concentration of each yfCBS construct were determined via UV spectroscopy $\left(\varepsilon_{280}=55,900 \mathrm{M}^{-1} \mathrm{~cm}^{-1}\right)$ (Jhee et al. 2000a). 


\subsection{Expression and Purification of htCBS Mutants}

All htCBS constructs were expressed and purified using the protocol reported by Belew et al. (2009). Three 2.8-L Fernbach flasks, each containing 1L of LB media, 75 $\mathrm{mg} / \mathrm{L} \delta$-aminolevulinic acid ( $\delta$-ALA) and $100 \mu \mathrm{g} / \mathrm{mL}$ ampicillin, were inoculated (1:50 ratio) with an overnight culture of the $E$. coli strain ER1821 containing the htCBS construct. These cultures were grown at $30^{\circ} \mathrm{C}$ to $\mathrm{OD}_{600}=0.5$, induced with $0.2 \mathrm{mM} \mathrm{IPTG}$ and incubated for an additional 16 hours. The cells were harvested via centrifugation at $5000 \mathrm{RPM}$ at $4^{\circ} \mathrm{C}$ for 10 minutes. The resulting pellets were resuspended with $0.85 \%$ $\mathrm{NaCl}$, centrifuged at $5000 \mathrm{RPM}$ for 15 minutes and the pellets were stored at $-80^{\circ} \mathrm{C}$.

The cells were resuspended and purified as described for yfCBS, with the exceptions that a $2 \mathrm{~mL}$ Ni-NTA column and a $200 \mathrm{~mL}$ linear gradient of $10-300 \mathrm{mM}$ imidazole were employed. The protein concentration of each htCBS construct were determined via $\mathrm{UV}$ spectroscopy $\left(\varepsilon_{280}=59,243 \mathrm{M}^{-1} \mathrm{~cm}^{-1}\right)$.

\subsection{Spectroscopic Characterization of htCBS}

The UV-Visible spectra of the CBS enzymes were recorded using an Agilent diode array model $8453 \mathrm{UV}$-visible spectrophotometer at $25^{\circ} \mathrm{C}$. All htCBS variants were diluted in $50 \mathrm{mM}$ Tris buffer ( $\mathrm{pH} 8.6$ ) to a final concentration of $4 \mu \mathrm{M}$ and their respective spectra were collected. A truncated yeast CBS enzyme (ytCBS), which was diluted to a final concentration of $4 \mu \mathrm{M}$, was used to demonstrate the masking of PLP by the heme cofactor. 


\subsection{Enzyme Assays}

Enzyme activity was measured in a total volume of $100 \mu \mathrm{L}$ at $25^{\circ} \mathrm{C}$ with a Spectramax 390 spectrophotometer (Molecular Devices). The assay buffer consisted of $50 \mathrm{mM}$ Tris $(\mathrm{pH} 8.6)$ and $20 \mu \mathrm{M}$ PLP. A background rate, for all components except the CBS enzyme, was recorded for each sample before initiating the reaction via the addition of CBS. The data were fit using KaleidaGraph (Synergy Software).

\subsubsection{Hydrolysis of L-Cystathionine by yfCBS}

The reverse-physiological hydrolysis of L-Cth to $\mathrm{L}-\mathrm{Ser}$ and L-Hcys was detected via the reaction of 5,5'-dithiobis-(2-nitrobenzoic acid) (DTNB) with the thiol group of the product (Aitken and Kirsch, 2003). L $^{-h o m o c y s t e i n e ~ r e a c t s ~ w i t h ~ D T N B ~ t o ~ f o r m ~ a ~}$ homocysteine/TNB- (3-thio-6-nitrobenzoate) mixed disulfide, with concomitant formation of $\mathrm{TNB}^{-}\left(\varepsilon_{412}=13,600 \mathrm{M}^{-1} \mathrm{~cm}^{-1}\right)$ (Figure 2.1). Reactions were carried out in assay buffer containing $2 \mathrm{mM}$ DTNB, 0.004-6.2 $\mathrm{mM}$ L-Cth and 0.675-2.175 $\mu \mathrm{M}$ yfCBS, depending on the activity of the variant. The data were fitted to the Michaelis-Menten equation (equation 1) to determine $k_{c a t}$ and $K_{m}$ while $k_{c a t} / K_{m}$ was calculated independently using equation 2:

$$
\frac{v}{[E]}=\frac{k_{c a t}[S]}{K_{m}+[S]}
$$

$$
\frac{v}{[E]}=\frac{k_{c a t} / K_{m}[S]}{1+[S] / K_{m}}
$$




\section{Cystathionine $\beta$-synthase}

(CBS)

Figure 2.1: DNTB assay utilized to measure the reverse-physiological hydrolysis of L-Cth by CBS (Aitken and Kirsch, 2003). 


\subsubsection{Condensation of L-Serine and L-Homocysteine by $h t C B S$}

The CBL-LDH continuous assay was developed to monitor the condensation of $\mathrm{L}^{-}$ Ser and L-Hcys, yielding L-Cth (Aitken and Kirsch, 2003). The L-Cth product is cleaved by eCBL, producing L-Hcys, $\mathrm{NH}_{3}{ }^{+}$and pyruvate, which is reduced to lactate by $\mathrm{LDH}$, with the concomitant oxidation of NADH $\left(\varepsilon_{340}=6,200 \mathrm{M}^{-1} \mathrm{~cm}^{-1}\right)$ to $\mathrm{NAD}^{+}$(Figure 2.2). Reactions were completed in assay buffer containing $1.5 \mathrm{mM} \mathrm{NADH,} 1 \mu \mathrm{M}$ CBL, 0.5 $\mu \mathrm{M}$ LDH, $0.2-36 \mathrm{mM}_{\mathrm{L}}$-Ser and $2 \mathrm{mM}$ Hcys as well as 1.23-4.11 $\mu \mathrm{M}$ htCBS. The data were fitted to the Michaelis-Menten equation while the $k_{c a t} / K_{m}$ values of the L-Ser substrate was calculated independently using equation 2 . 


\section{Cystathionine $\beta$-synthase}

(CBS)

Figure 2.2: The assay employed to determine the $\beta$-replacement activity of CBS, via the use of CBL and LDH coupling enzymes (Aitken and Kirsch, 2003). 


\section{RESULTS}

\subsection{The Construction and Purification of the Site-Directed Variants}

The yfCBS and htCBS variants were produced by overlap extension PCR (Figure 3.1). The amplicons introduced between the NdeI and PstI (hCBS) or NdeI and HindIII (yCBS) sites of the pTrc3 expressions vector and the resulting constructs were used to transform E. coli strain ER1821. The wild-type and site-directed variants were purified by Ni-NTA affinity chromatography and their purity was assessed via SDS-PAGE (Figures $3.2 \& 3.3$ ). The yields were between $0.33-1.98 \mathrm{mg}$ and $0.41-4.44 \mathrm{mg}$ per $1 \mathrm{~L}$ culture for the yfCBS and htCBS variants, respectively (Table 3.1)

\subsection{Kinetic Characterization of the yfCBS Variants}

The kinetic parameters of the S323A and Y324F variants are similar to those of wild-type yfCBS (Table 3.2). The $k_{c a t} / K_{m R}{ }^{\mathrm{L}-\mathrm{Cth}}$ of the S323A variant is decreased only $\sim 6$ fold, corresponding to an equivalent increase in $K_{m R}{ }^{L-C t h}$. The Y324F substitution results in a reduction in $k_{c a t R}$ and an increase in $K_{m R}{ }^{L-C t h}$ of a similar magnitude. No activity was detected for yfCBS-D319A. 


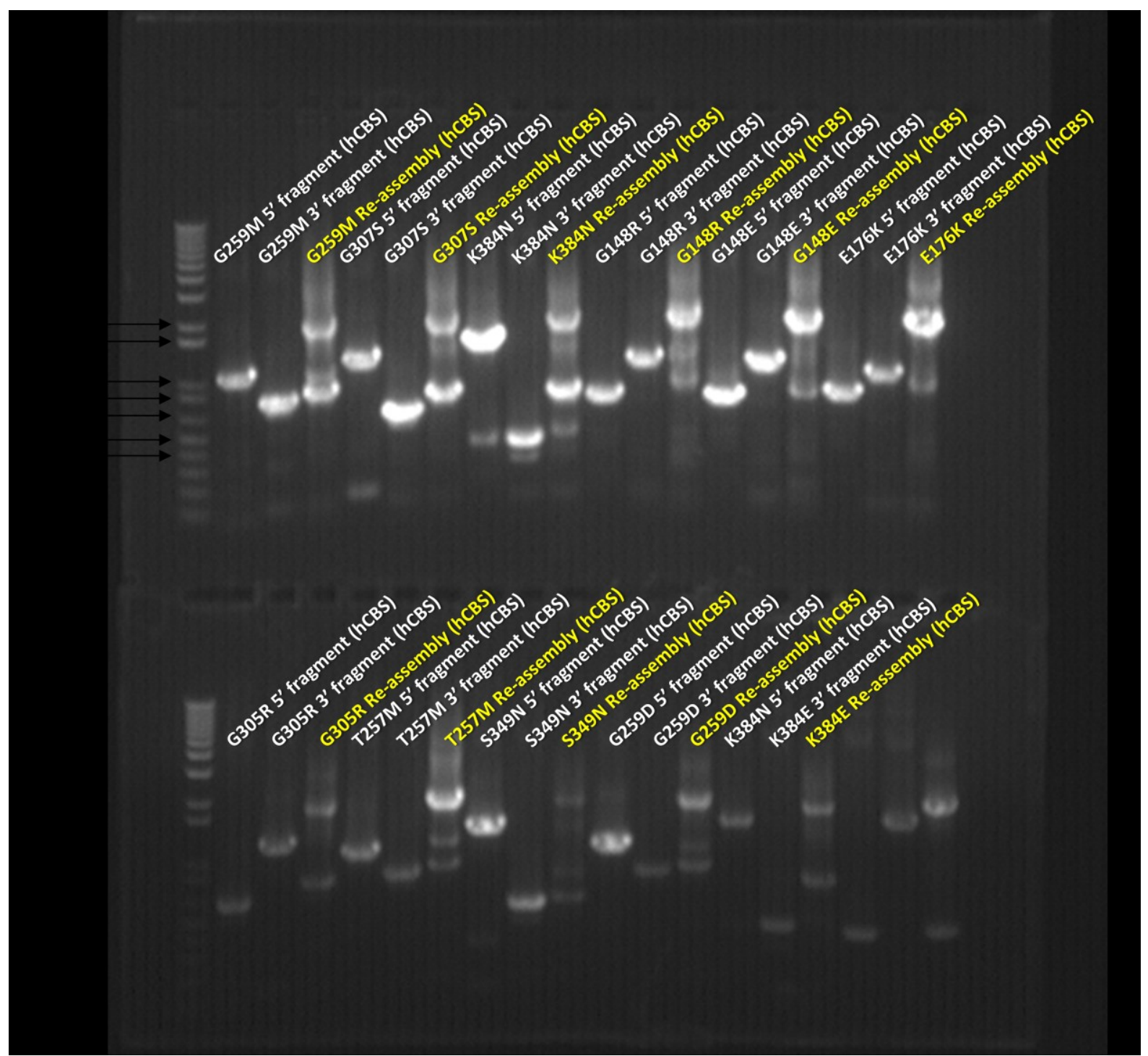

Figure 3.1: Agarose gel of PCR amplicons produced for the construction of the htCBS site-directed mutants via overlap-extension PCR. Each pair of 5' (Left Rxn; White) and 3' (Right Rxn; White) amplicons, which overlap by the mutagenic primer sequence, were combined and amplified with oligonucleotide primers flanking the start and stop codons to produce the reassembled htCBS sequence with the desired mutation (Yellow). 


\section{yfCBS yfCBS yfCBS yfCBS WT S323A Y324F D319A}

\section{$\sim 63 \mathrm{kDa}$}

\section{$50 \mathrm{kDa}$}

Figure 3.2: A SDS-PAGE gel, stained with Comassie Blue, showing $4 \mu \mathrm{g}$ each of the wild-type and S323A, Y324F and D319A site-directed variants of yfCBS. The approximate sizes of protein bands are indicated. 


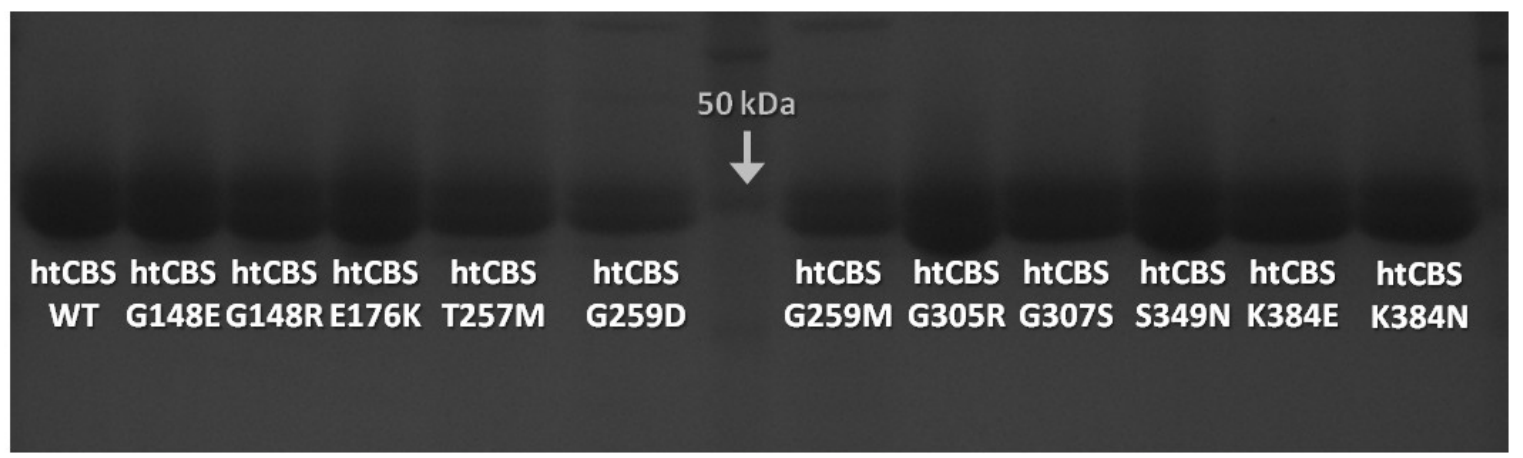

Figure 3.3: A SDS-PAGE gel, stained with Comassie Blue reagent, showing $4 \mu \mathrm{g}$ of the wild-type and site-directed variants of htCBS. The $50 \mathrm{kDa}$ protein marker band is indicated. 
Table 3.1: Protein yield of yfCBS and htCBS variants.

\begin{tabular}{||||c||c||}
\hline yfCBS Variant & Yield (mg/L) \\
\hline Wild-type & n.a. ${ }^{*}$ \\
\hline D319A & 0.33 \\
\hline S323A & 1.98 \\
\hline Y324F & 1.03 \\
\hline \hline htCBS Variant & Yield (mg/L) \\
\hline Wild-type & 4.44 \\
\hline G148E & 2.46 \\
\hline G148R & 1.64 \\
\hline E176K & 2.18 \\
\hline T257M & 0.68 \\
\hline G259D & 0.41 \\
\hline G259M & 0.67 \\
\hline G305R & 2.20 \\
\hline G307S & 2.79 \\
\hline S349N & 3.63 \\
\hline K384E & 3.39 \\
\hline K384N & 2.25 \\
\hline
\end{tabular}

*Wild-type yfCBS was purified by Sherwin Habibi and yield was not reported.

Table 3.2: Steady-state kinetics parameters of wild-type yfCBS and the D319A, S323A and Y324F variants for the reverse-physiological hydrolysis of L-cystathionine.

\begin{tabular}{||c|c|c|c||}
\hline \hline Enzyme & $\boldsymbol{k}_{\text {catR }}\left(\mathbf{s}^{-\mathbf{1}}\right)$ & $\mathbf{K}_{\mathbf{m} R}{ }^{\mathrm{L}-C t h} \mathbf{( m M )}$ & $\boldsymbol{k}_{\text {cat }} / \boldsymbol{K}_{\boldsymbol{m} \boldsymbol{R}^{\mathrm{L}-C t h}}\left(\mathbf{M}^{-\mathbf{1}} \mathbf{s}^{-\mathbf{1}}\right)$ \\
\hline wt yfCBS & $0.147 \pm 0.003$ & $0.088 \pm 0.008$ & $(1.7 \pm 0.1) \times 10^{3}$ \\
\hline S323A & $0.145 \pm 0.003$ & $0.557 \pm 0.005$ & $(0.26 \pm 0.02) \times 10^{3}$ \\
\hline Y324F & $0.071 \pm 0.002$ & $0.20 \pm 0.02$ & $(0.35 \pm 0.03) \times 10^{3}$ \\
\hline D319A & \multicolumn{3}{|c|}{ No Activity Detected } \\
\hline
\end{tabular}

*Kinetic measurements were determined at $25^{\circ} \mathrm{C}$ using a $50 \mathrm{mM}$ Tris buffer $(\mathrm{pH} 8.6)$ containing $20 \mu \mathrm{M}$ PLP, $2 \mathrm{mM}$ DTNB, 0.004-6.2 $\mathrm{mM}$ L-Cth and 0.675-2.175 $\mu \mathrm{M}$ yfCBS. Data were fit to Eqs. (1) and (2). 


\subsection{Kinetic Characterization of the htCBS Variants}

All of the homocystinuria-associated substitutions examined in this study impacted the catalytic efficiency of htCBS. A universal decrease in the turnover rate with respect to L-Ser $\left(k_{\text {cat }}{ }^{L-S e r}\right)$ was observed and ranges from 2.5 -fold, for htCBS-K384N, to 19-fold for htCBS- G307S (Table 3.3). The kinetic parameter least affected by the various substitutions is the Michaelis constant for $_{\mathrm{L}}$-serine, as the $K_{m}{ }^{L \text {-Ser }}$ of 9 of the 11 variants is within 3-fold of the wild-type value, while the substitution of residue K384 to either the negatively charged glutamate (K384E) or the polar residue asparagines (K384N) led to a approximately 4- and 5- fold reduction in their respective $K_{m}{ }^{L-S e r}$ values (Table 3.3).

\subsection{Spectral Characterization of the htCBS Wild-type Enzyme and Site-Directed}

\section{Variants}

The absorbance spectrum of the wild-type htCBS is characterized by a pair of absorbance peaks, of similar intensity, centred at 280 and $430 \mathrm{~nm}$ (Figure 3.4). The peak at $280 \mathrm{~nm}$ corresponds to the side chains of aromatic amino acids tryptophan, tyrosine and phenylalanine of the htCBS apoenzyme, while the $430 \mathrm{~nm}$ peak is attributable to the Soret absorbance of the heme cofactor. The PLP cofactor absorbs in the same region of the spectrum as the Soret band, but is masked by the 7 -fold greater absorbance intensity of the latter (Figure 3.4).

The majority of the htCBS site-directed variants exhibit spectral profiles similar to the wild-type enzyme, with the exception of shifts in the intensity of the 280 and 430 $\mathrm{nm}$ peaks (Figure 3.5A). In contrast, the Soret absorbance of three variants (T257M, 
$\mathrm{G} 259 \mathrm{D} / \mathrm{M}$ ) is blue-shifted, compared to the wild-type enzyme (Figure 3.5B). The Soret peaks of both G259 variants are shifted $\sim 25 \mathrm{~nm}$, from 430 to $405 \mathrm{~nm}$, while that of htCBS-T257M is only slightly shifted to $428 \mathrm{~nm}$.

The ratio between the absorbances of the Soret and aromatic peaks $\left(\mathrm{A}_{430 / 280}\right)$ has previously been used as a qualitative measure of heme binding to hCBS (Majtan et al., 2010). In this study, $\mathrm{A}_{430 / 280}$ was used to examine the effect of each site-directed substitution on heme saturation. The wild-type truncated hCBS enzyme, lacking the Cterminal, regulatory domain, possesses an identical $\mathrm{A}_{430 / 280}$ to that reported for the fulllength enzyme (1.10; Majtan et al., 2010). Eight variants exhibit $\mathrm{A}_{430 / 280}$ ratios that range between 0.73 (htCBS-S349N) and 0.90 (htCBS-G148E) (Figure 3.5A). Substitution of residues involved in coordination of the phosphate group of PLP (T257M, G259D/M) show the largest reductions in the $\mathrm{A}_{430 / 280}$ ratio (Figure 3.5B). The ratios of the $\mathrm{T} 257 \mathrm{M}$, G259D and G259M variants were reduced by 2-3.5-fold in addition to the observed blueshift of the absorbance maximum. 
Table 3.3: Steady-state kinetics parameters for the condensation of L-serine (L-Ser) and L-homocysteine (L-Hcys) by wild-type htCBS and homocystinuria-associated variants.

\section{L-Serine + L-Homocysteine $\rightarrow$ L-Cystathionine}

\begin{tabular}{|l||c|c|c|c|c|c||}
\hline \hline Kinetic Parameters & wt-htCBS & G148E & G148R & E176K & T257M & G259D \\
\hline${ }^{a} \mathrm{k}_{\mathrm{cat}}{ }^{\mathrm{L}-S e r}\left(\mathrm{~s}^{-1}\right)$ & $1.69 \pm 0.03$ & $0.195 \pm 0.004$ & $0.17 \pm 0.01$ & $0.30 \pm 0.02$ & $0.25 \pm 0.01$ & $0.36 \pm 0.01$ \\
\hline${ }^{\mathrm{a}} \mathrm{K}_{\mathrm{m}}{ }^{\mathrm{L}-S e r}(\mathrm{mM})$ & $3.0 \pm 0.2$ & $6.2 \pm 0.5$ & $9 \pm 1$ & $8.4 \pm 1.5$ & $7 \pm 1$ & $4.6 \pm 0.5$ \\
\hline${ }^{\mathrm{a}} \mathrm{k}_{\mathrm{cat}} / \mathrm{K}_{\mathrm{m}}{ }^{\mathrm{L}-\mathrm{Ser}}\left(\mathrm{M}^{-1} \mathrm{~s}^{-1}\right)$ & $570 \pm 40$ & $32 \pm 2$ & $19 \pm 1$ & $35 \pm 4$ & $34 \pm 4$ & $78 \pm 7$ \\
\hline
\end{tabular}

\begin{tabular}{|l||c|c|c|c|c|c||}
\hline \multirow{2}{*}{ Kinetic Parameters } & G259M & G305R & G307S & S349N & K384E & K384N \\
\hline${ }^{a} \mathrm{k}_{\text {cat }}{ }^{\mathrm{L}-S e r}\left(\mathrm{~s}^{-1}\right)$ & $0.33 \pm 0.02$ & $0.15 \pm 0.01$ & $0.09 \pm 0.01$ & $0.117 \pm 0.004$ & $0.103 \pm 0.003$ & $0.69 \pm 0.03$ \\
\hline${ }^{\mathrm{a}} \mathrm{K}_{\mathrm{m}}{ }^{\mathrm{L}-S e r}(\mathrm{mM})$ & $8 \pm 1$ & $7 \pm 1$ & $6.1 \pm 0.9$ & $4.7 \pm 0.6$ & $11 \pm 1$ & $18 \pm 1$ \\
\hline${ }^{\mathrm{a}} \mathrm{k}_{\mathrm{cat}} / \mathrm{K}_{\mathrm{m}}{ }^{\mathrm{L}-S e r}\left(\mathrm{M}^{-1} \mathrm{~s}^{-1}\right)$ & $40 \pm 4$ & $23 \pm 2$ & $15 \pm 1$ & $25 \pm 3$ & $10 \pm 1$ & $39 \pm 2$ \\
\hline
\end{tabular}

*Kinetic measurements were carried out in $50 \mathrm{mM}$ Tris ( $\mathrm{pH} 8.6$ ) containing $20 \mu \mathrm{M}$ PLP, $1.5 \mathrm{mM}$ NADH, $1 \mu \mathrm{M} \mathrm{CBL}, 0.5 \mu \mathrm{M}$ LDH, $0.2-36 \mathrm{mM}_{\mathrm{L}}$-Ser with $2 \mathrm{mM}_{\mathrm{L}}$-Hcys, and $1.23-4.11 \mu \mathrm{M}$ htCBS at $25^{\circ} \mathrm{C}$.

${ }^{a}$ Data were fitted to Eqs. (1) and (2) 


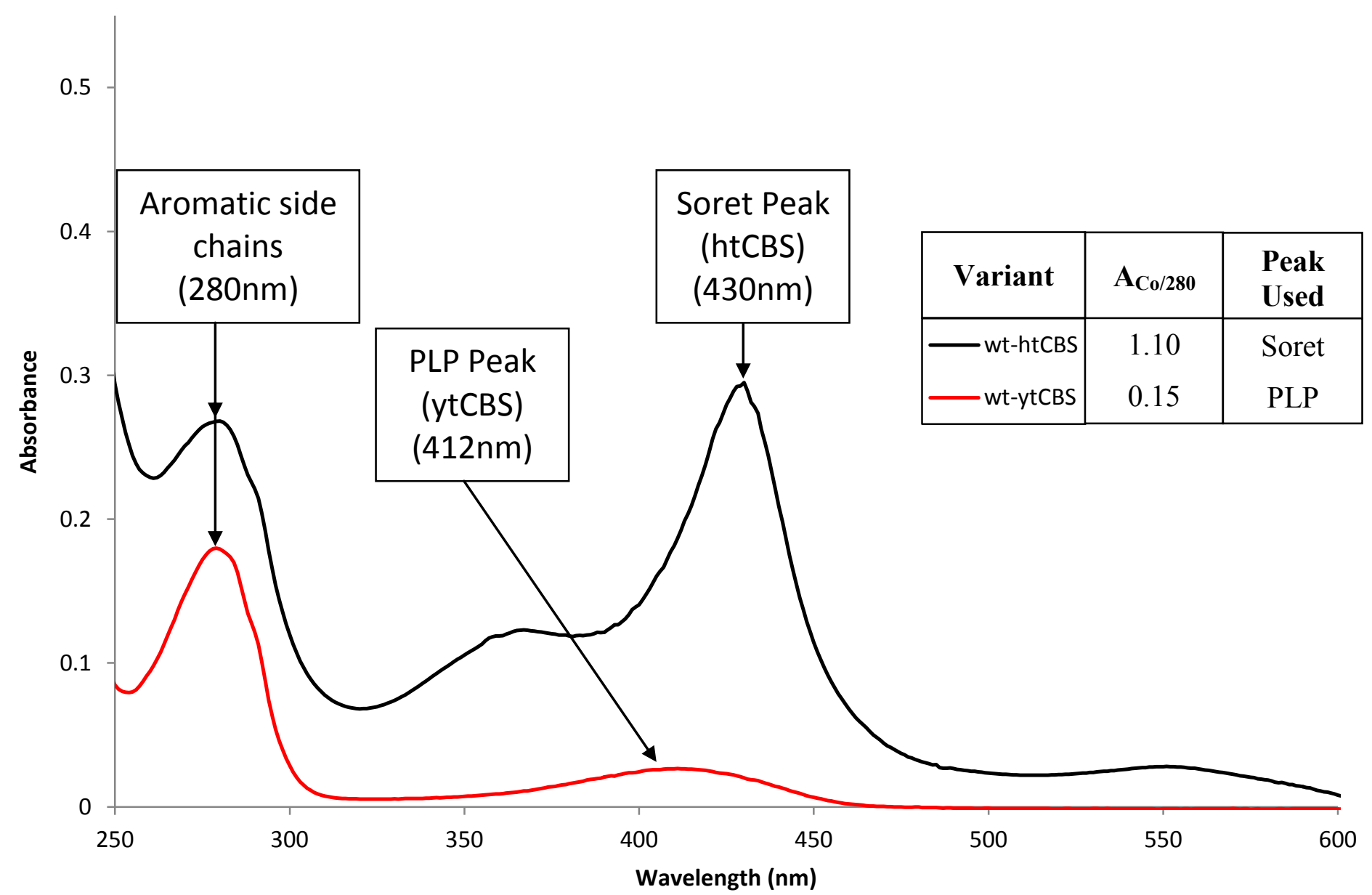

Figure 3.4: UV-Visible spectra of the wild-type htCBS (black) and ytCBS (red) enzymes. Comparison of the spectra of the two enzymes demonstrates that the Soret absorbance of the heme cofactor masks that of the PLP in the 400-460 $\mathrm{nm}$ region. 

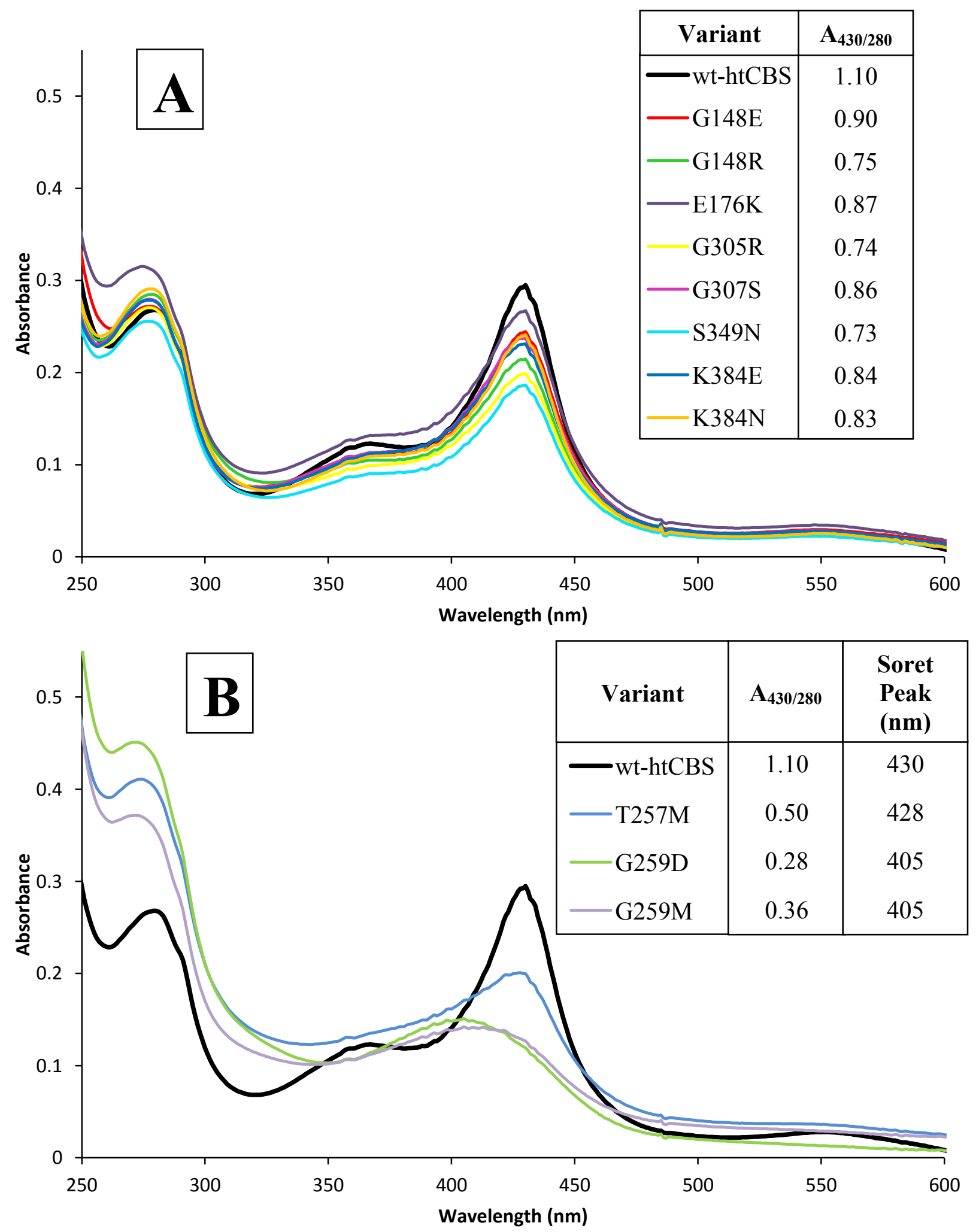

Figure 3.5: UV-Visible spectra of the purified htCBS variants examined in this study. Comparison between spectra of the wild-type wt-htCBS enzyme and (A) the 8 variants (G148E/R, E176K, G305R, G307S, S349N, K384E/N) similar to the wild-type enzyme, and (B) the T257M and G259D/M variants. The ratio of the absorbance at 430 and 280 $\mathrm{nm}$ peaks provides an indication of heme saturation. 


\section{DISCUSSION}

As the first enzyme in the reverse transsulfuration pathway, CBS catalyzes the condensation of the branch-point metabolite L-Hcys with L-Ser to produce L-Cth. The flow of metabolites from the L-Hcys branch point has been well-studied as it is associated with homocystinuria (Mudd et al., 2001). However, relatively few studies have probed the structure-function relationships that underlie the many $(>100)$ unique diseaseassociated alleles of CBS. In this study, the effect of a series of homocystinuriaassociated substitutions, located in proximity to the active site, on the kinetic parameters of the enzyme was investigated. The results enhance our understanding of the molecular basis of homocystinuria and contribute to a body of knowledge that can form the basis for the development of novel therapeutics.

The common theme of the two studies that comprise this thesis is exploration of the hydrogen bonding networks of the active site. The role of three residues (D319, S323 and Y324) that interact with N84, which tethers the O3' atom of the PLP cofactor, in the active-site of the model yeast CBS enzyme was probed. A series of 11 homocystinuriaassociated mutations (G148E/R, E176K, T257M, G259D/M, G305R, G307S, S349N, $\mathrm{K} 384 \mathrm{E} / \mathrm{N}$ ), situated in proximity to the active-site of human CBS were also investigated.

\subsection{The yfCBS Variants (D319A, S323A \& Y324F)}

Residues D319, S323 and Y324 are located at the junction of the catalytic domain and the linker segment that connects the catalytic core and the regulatory domain of yCBS. These residues are within hydrogen bonding distance of N84 (N149 in hCBS numbering), which forms a ketoenamine-stabilizing hydrogen bond with the O3' position of the PLP cofactor (Figure 4.1) (Lodha et al., 2010; Weeks et al., 2009; Koutmos et al., 
2010). Substitution of N84 with asparatate in ytCBS causes a reduction in $k_{\text {cat } F} / K_{m F}{ }^{L-S e r}$ of four orders of magnitude and the development of a marginal $\beta$-elimination activity (Lodha et al., 2010). A set of conservative substitutions that remove the hydrogen bonding capacity of the side chains of the targeted residues (D319A, S323A and Y324F) was constructed to examine their effect on the activity of the model yeast enzyme. A schematic representation of the yCBS active site is presented in Figure 4.1 in which the targeted S323, Y324 and D319 are shown, with respect to key residues involved in substrate binding and catalysis. The full-length yfCBS enzyme was employed for this study because of the location of the 3 residues in proximity to the linker and regulatory domain.

Analysis of the structures of hCBS and dCBS suggests that the side-chain carboxylate moiety of D319 forms a hydrogen bond with the amide nitrogen of the N84 side chain (Figure 4.1). The lack of detectable activity observed for the yfCBS-D319A variant, which differs by only the missing side-chain carboxylate group of D319, may be due to improper positioning of the PLP cofactor. The negatively charged carboxylate group may also play an important role in modulating the strength of the hydrogen bond between N84 and O3' of the cofactor, thereby influencing electron distribution in the ring of the cofactor during catalysis. Loss of this influence and repositioning of N84, resulting from removal of the D319 carboxylate group, may thereby disturb the subtle active-site dynamics required for catalysis. This residue is conserved across all known CBS enzymes including hCBS (D376) and dCBS (D345), providing indirect evidence of the requirement for a negatively charged and/or hydrogen bonding side chain at position 319 (Chang and Vining, 2002, Kabil et al., 2011; Oyenarte et al., 2012). 
The side-chains of residues D319 and Y324 are connected by a hydrogen bond, raising the possibility that Y324 may play an indirect role (D319-N84-mediated) in positioning the cofactor and determining active-site architecture (Figure 4.1). The removal of the Y324 side-chain hydroxyl moiety, through a tyrosine-to-phenylalanine substitution, results in a negligible effect on the function of the enzyme as both $K_{m}{ }^{L-C t h}$ and $k_{c a t}$ are within $\sim 2$-fold of the wild-type enzyme. This demonstrates that the hydrogen bonding capacity of the Y324 side chain is not a key determinant of active-site architecture or cofactor positioning.

Residue S323, like D319, is situated within hydrogen bonding distance of the side-chain amide moiety of N84 (Figure 4.1). The $K_{m}{ }^{L-C t h}$ of yfCBS-S323A is increased $\sim 6$-fold, while $k_{\text {cat }}$ is unchanged (Table 3.2). This suggests that while the cofactor positioning within the active site is not changed by removal of the hydrogen bond between S323 and N84, the binding site of the L-Ser substrate may be subtly altered. Interestingly, S323 is a yeast-specific residue as its equivalent in all other known CBS enzymes is an asparagine residue. 


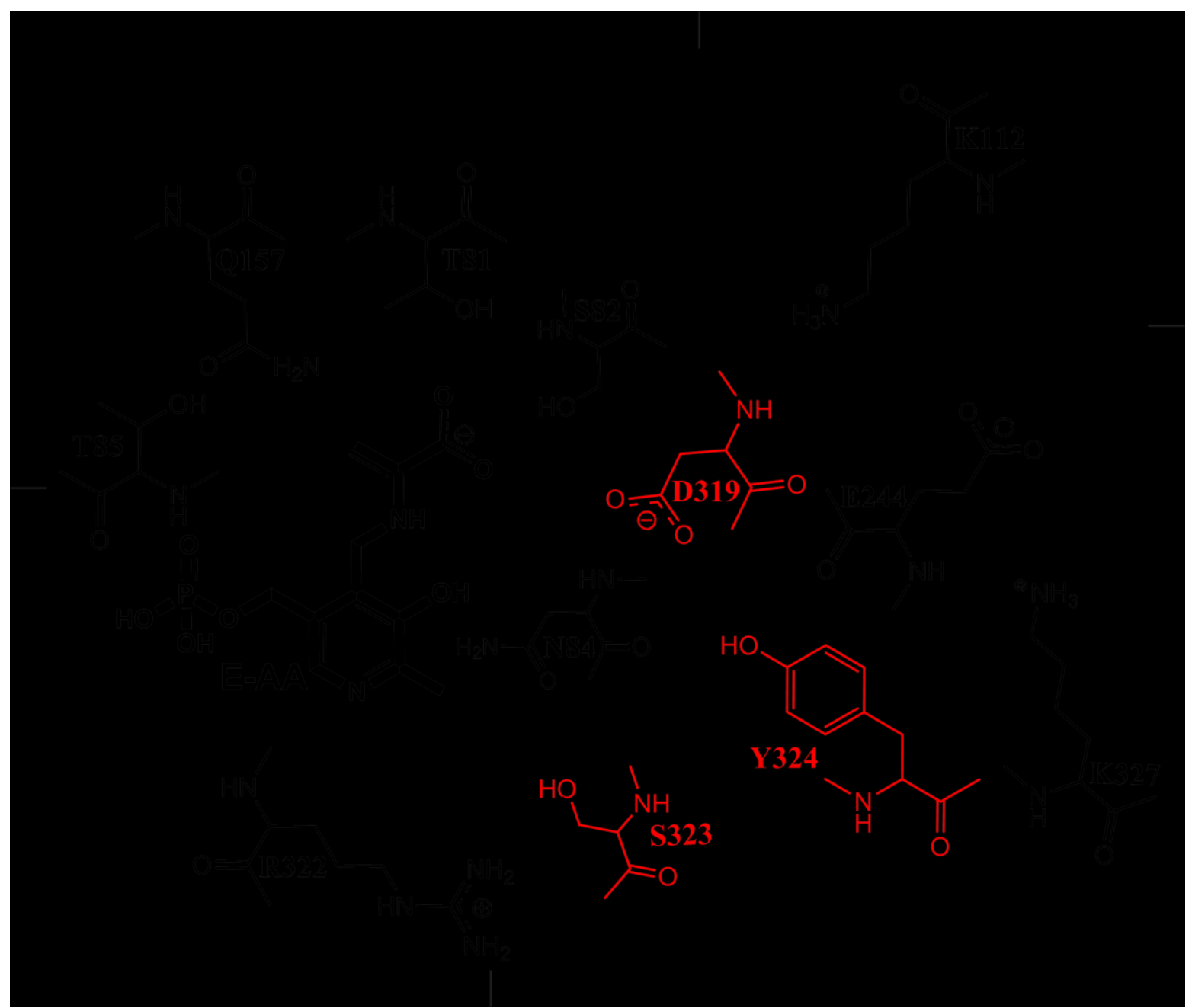

Figure 4.1: Proposed active site contacts in the yCBS-aminoacrylate complex. The putative hydrogen bond distances (represented by dotted lines; $<3.2 \AA$ between heteroatoms) are based on interactions observed in the structure of dCBS (Koutmos et al., 2010; Lodha et al., 2010). Residues numbering corresponds to yCBS. 


\subsection{The Truncated Human CBS Enzyme}

Belew et al. (2009) determined the kinetic parameters of the 6-His tagged, fulllength hCBS (hfCBS) and the affinity-tagged truncated human enzyme constructed for this study was compared to this version of the full-length wild-type enzyme. In keeping with reports that removal of the C-terminal domain, which blocks substrate access to the active-site, increases enzyme activity (Evande et al., 2002; Ereno-Orbea et al., 2013a), the $k_{\text {cat }}^{L-S e r}$ of htCBS are 1.4-fold higher than the full-length enzyme $\left(k_{\text {cat }}{ }^{L-S e r}=1.69 \pm\right.$ $\left.0.06 \mathrm{~s}^{-1}\right)$. The observed minor changes in $K_{m}{ }^{L-S e r}$ may be due to slight differences in the architecture of the active site resulting from loss interactions between residues of the regulatory and catalytic domains, in the context of the truncated enzyme. The effect of 11 homocystinuria-associated mutations, of residues located in, or close to, the active-site of htCBS, on activity was investigated: G148E/R, E176K, T257M, G259D/M, G305R, G307S, S349N and K384E/N.

\subsubsection{Residue $G 148$}

G148 is situated in the middle of the mobile loop (T146-S147-G148-N149-T150) which undergoes a conformational shift upon binding of the L-Ser substrate to the active site. The hydrogen bond between the backbone amide of G148 and the hydroxyl group of T146 may aid in positioning of the latter, enabling formation of a hydrogen bond with the L-Ser substrate (Figure 4.2). Substitution of G148 with either a negatively-charged (G148E) or a positively-charged residue (G148R) is detrimental to the function of the enzyme. Both variants result in a decrease of approximately 9-fold in $k_{c a t}^{L-S e r}$ and a 2-3fold increase in $K_{m}{ }^{L-S e r}$ (Table 3.3). The hydrogen side chain of G148 likely provides 
flexibility required by the mobile loop as it moves between the open and closed conformations of the active site. Substitution of this residue with one possessing a bulky side chain, such as glutamate or arginine, would be expected to limit the flexibility of the loop and limit its range of motion compared to the wild-type enzyme.

The G148R variant has been classified as nonresponsive to vitamin $\mathrm{B}_{6}$ supplementation and the clinical responsiveness of the G148E variant has not been assessed (Kraus et al., 1999). Based on comparable effects to the kinetic parameters of both variants, it may be inferred that a patient with the G148E phenotype is also likely to be $\mathrm{B}_{6}$-nonresponsive. The presence of the bulky side chain at position 148 may interfere with proper substrate positioning rather than cofactor binding, such that the effect of the substitution is not alleviated by $\mathrm{B}_{6}$ supplementation. The observed changes in the turnover of the htCBS-G148E/R variants would be expected to reduce L-Hcys flux through the reverse transsulfuration pathway, thereby leading to its observed accumulation in homocystinuric patients. 


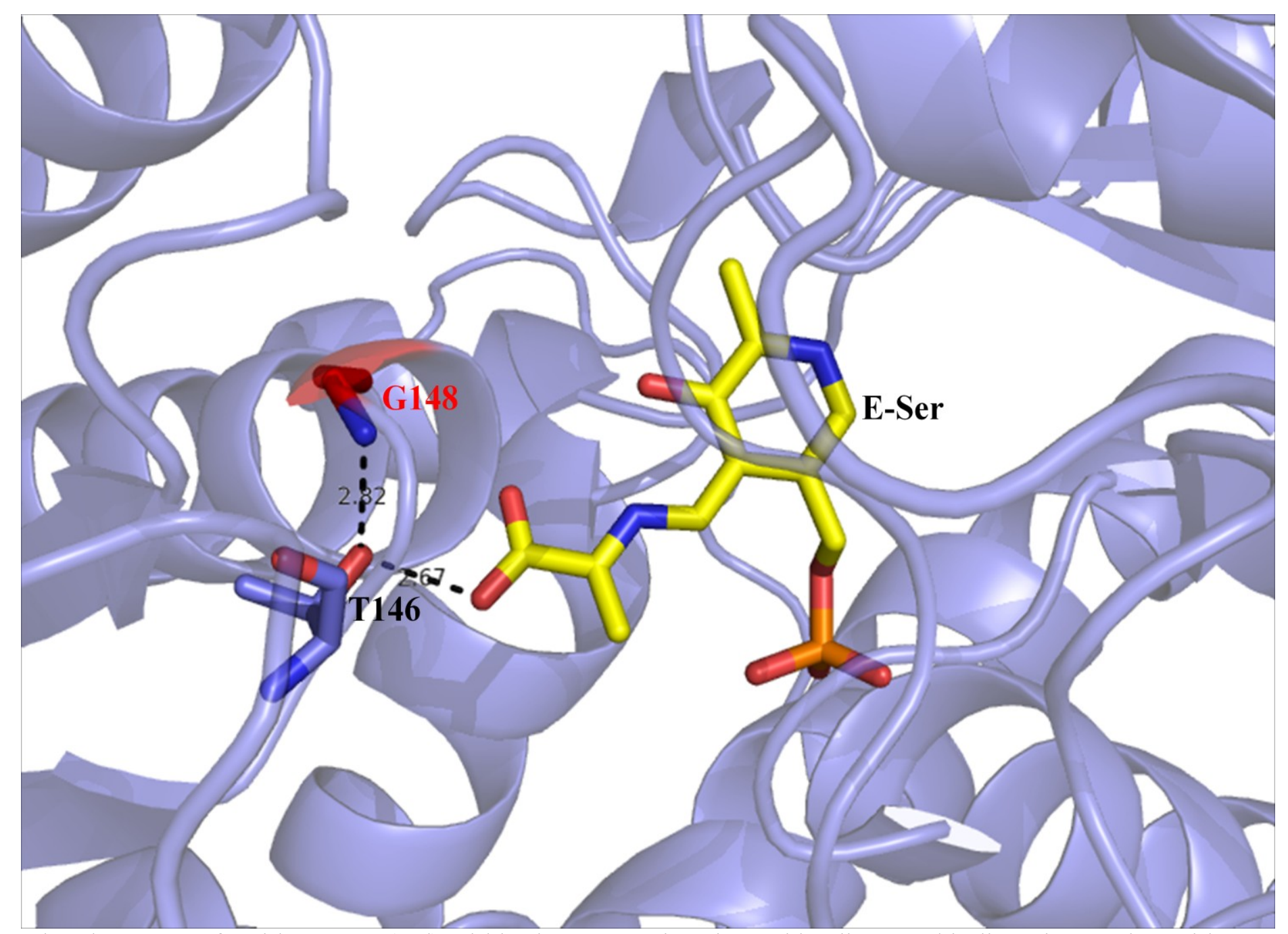

Figure 4.2: The placement of residue G148 (red) within the CBS active site and its direct and indirect interaction with T146 (pale blue) and the external aldimine (E-Ser; Yellow), respectively. Proposed hydrogen bond distances are indicated and shown in yellow (Koutmos et al., 2010). 


\subsubsection{Residues E176 and K384}

Residues E176 and K384 are members of the K177 hydrogen bonding network, which also includes E304, T383 and S147 (Figure 4.3). The network is responsible for bridging the mobile loop of the active site with the C-terminal portion of the catalytic core. The segments connected by this hydrogen bonding network (the mobile loop as well as loops 296-316 and 374-388) constitute a large portion of the surface of the active site. Lodha and colleagues showed that conservative substitution of the residue corresponding to $\mathrm{K} 177$ in ytCBS (K112) with arginine produces a 471-fold reduction in catalytic efficiency of L-Ser, thereby illustrating the importance of this network in maintaining proper active site architecture (Lodha et al., 2009).

Charge-reversal substitution of position 176 (E176K) produces a 3-fold increase in $K_{m}{ }^{L-S e r}$ and a 6-fold decrease of the turnover rate. Substituting the negatively charged carboxylate group of E176 with a positively charged lysine would not necessarily eliminate the E176-T326 hydrogen bond, but may cause subtle changes in active-site architecture or communication with the regulatory domain, not present in htCBS (Figure 4.3). Hnizda et al. (2012) showed that the E176K variant in the full-length sequence was more susceptible to protein cleavage than the other variants examined in their study, suggesting that the effect of this $\mathrm{B}_{6}$-unresponsive substitution may prevent the enzyme in adopting a stably folded structure (Kraus et al., 1999; Hnizda et al., 2012).

The effect of substituting the lysine at position 384 with glutamate (K384E) was more detrimental to htCBS turnover than a replacement with asparagine (K384N) evident by the 7 -fold increase in the $k_{\text {cat }}{ }^{L-S e r}$ of K384N compared to K384E. Although the asparagine substitution removes the positive charge of the native lysine side chain and the 
glutamate substitution results in a charge reversal, both have a similar effect of reducing the size of the residue at position 384, thereby repositioning the hydrogen bonding component of the side chain. Surprisingly, individuals with the K384E phenotype responded to vitamin $\mathrm{B}_{6}$ treatment while those with the $\mathrm{K} 384 \mathrm{~N}$ variant were only partially responsive, as total homocysteine concentration remained $>50 \mu \mathrm{M}$ following to vitamin $\mathrm{B}_{6}$ treatment (Kraus et al., 1999). This may indicate that the presence of excess PLP shifts the equilibrium of active-site conformations of the K384E, and to a lesser extent $\mathrm{K} 384 \mathrm{~N}$ variant, in favor on one that is catalytically productive, thereby alleviating the L-Hcys buildup in individuals expressing either genotype. 


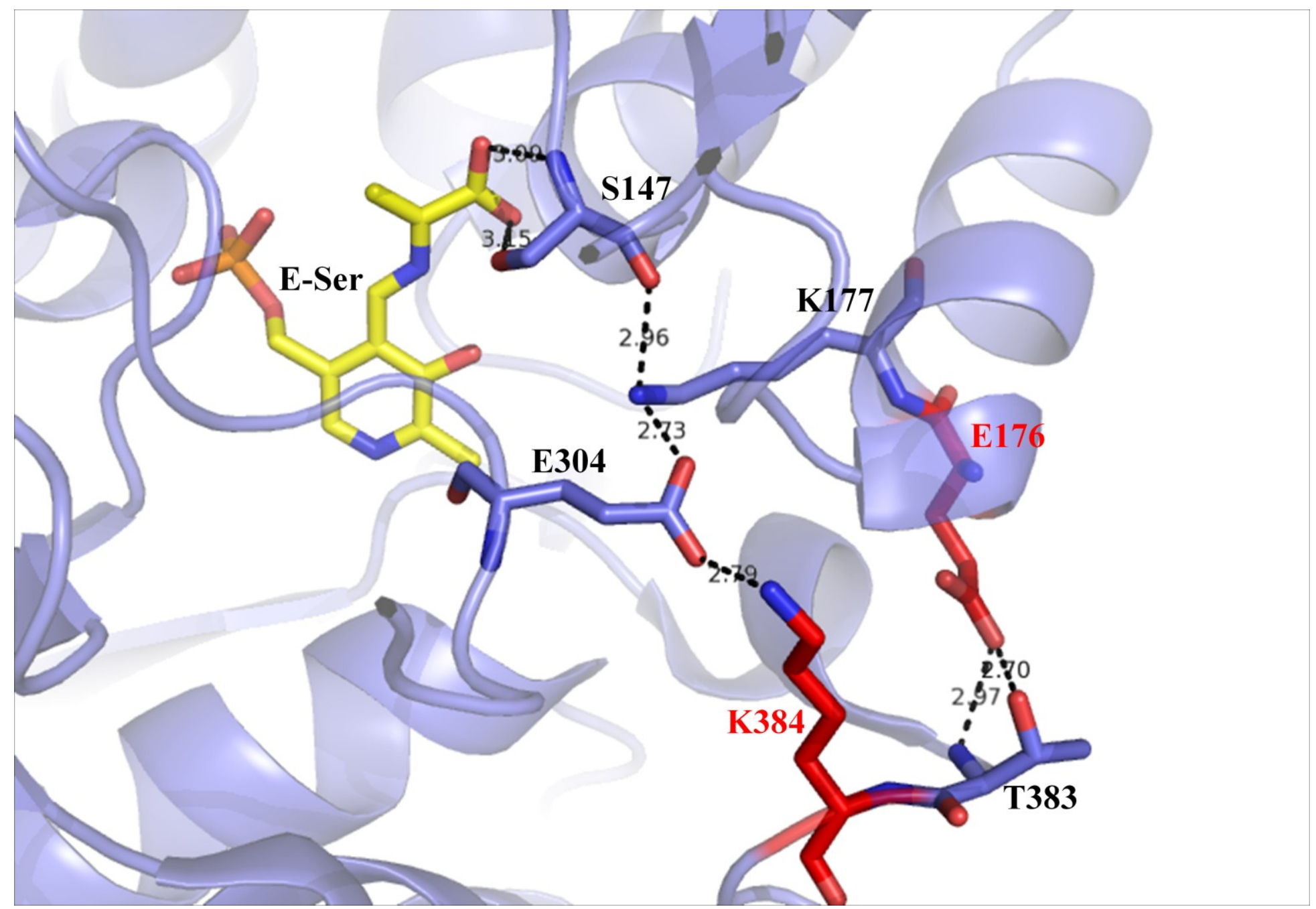

Figure 4.3: The location of the K177 hydrogen bonding network to which K384 and E176 are members of said group. The putative roles of K384 and E176 (red) within the network as well as the network's interaction with S147 in the closed conformation of CBS are shown (E-Ser; Yellow). Proposed hydrogen bond distances are indicated and shown in yellow (Koutmos et al., 2010). 


\subsubsection{Residues $T 257$ \& G259}

Residues T257 and G259 are a part of a conserved glycine rich loop (G256-T257G258-G259-T260) that forms multiple hydrogen bonds with the phosphate moiety of PLP and plays an important role in anchoring the cofactor within the active site (Figure 4.4). While G259 does not directly interact with the cofactor, its backbone amide moiety is within hydrogen bonding distance of the hydroxyl side-chain group of T257. Therefore, G259 may play an indirect role by positioning T257 to optimally align the latter to hydrogen bond with the phosphate group of the cofactor (Meier et al., 2001; Koutmos et al., 2010).

Replacement of T257 with methionine removes the hydrogen bonding capacity of this residue and increases the bulk of the side chain. Therefore, the observed 7 -fold decrease in the $k_{\text {cat }}{ }^{L-S e r}$ of T257M is likely due to a shift in the position of the cofactor within the active site, offering an explanation for this variant's unresponsiveness to vitamin B6 (Kraus et al., 1999). A $2 \mathrm{~nm}$ blue-shift, compared to the $430 \mathrm{~nm}$ maximum of the Soret peak of wild-type wt-htCBS, and a 2-fold decrease in $\mathrm{A}_{430 / 280}$ are also observed for this variant. Ojha et al. (2002) observed a slight blue-shift of the Soret band of a H65R full-length variant to $424 \mathrm{~nm}$, similar to the T257M variant examined in this study. As the overall shape of the Soret peak remained intact, indicating that the six-coordinate state of heme is retained, Ojha et al. (2002) hypothesized that the slight shift in the Soret peak may be due to a replacement of $\mathrm{H} 65$ as the axial iron ligand of the heme cofactor by the proximal H67 (Figure 4.5). The similar characteristics of the Soret peaks of the H65R and $\mathrm{T} 257 \mathrm{M}$ variants, suggest that $\mathrm{H} 65-\mathrm{H} 67$ heme ligand switching may be the source of the altered spectral profile of htCBS-T257M. 


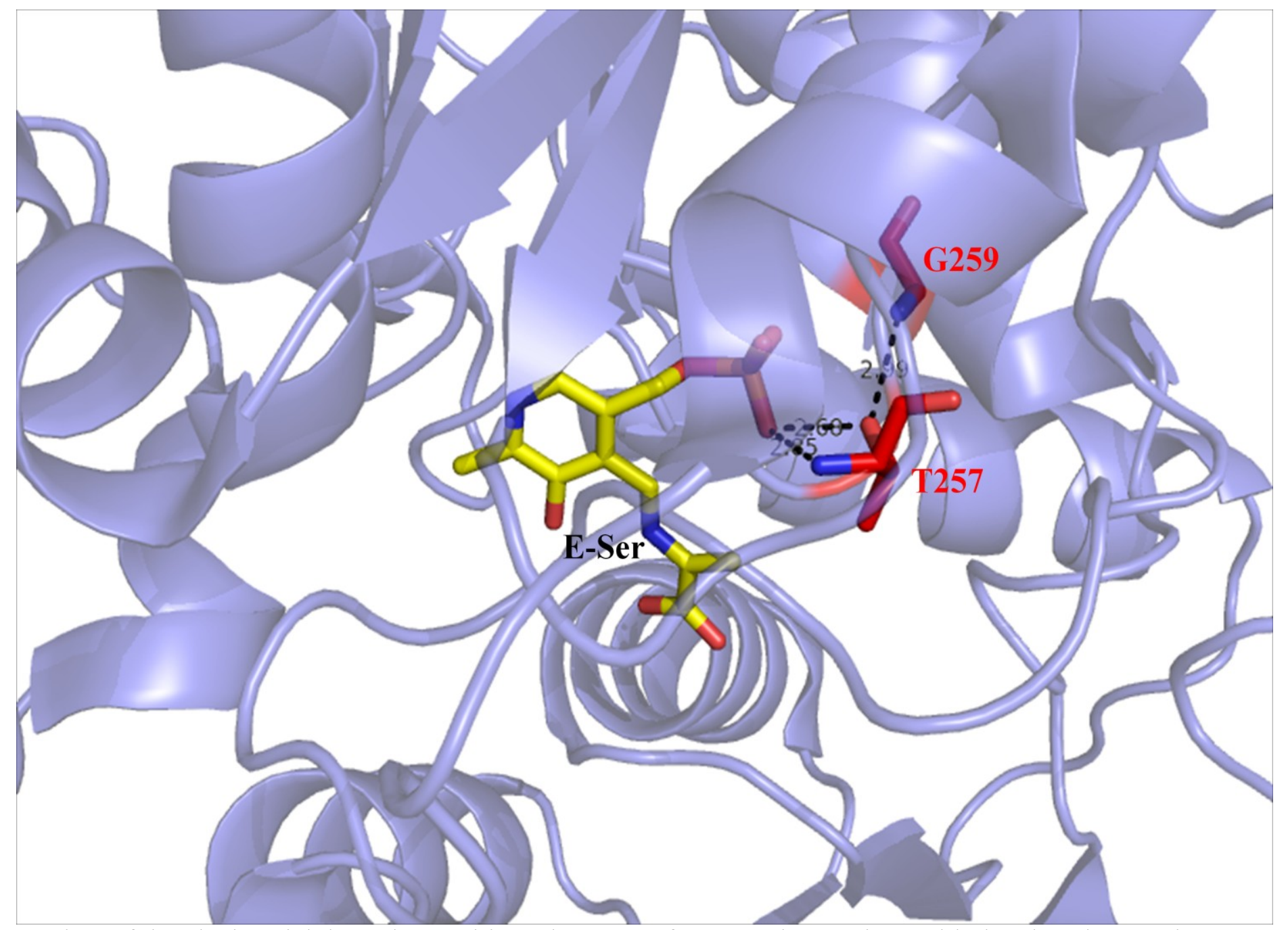

Figure 4.4: A view of the glycine-rich loop that positions the PLP cofactor via interactions with the phosphate moiety. Interactions between residues T257 and G259 (red) and the phosphate moiety of the aminoacrylate complex of the cofactor are shown (Yellow). Proposed hydrogen bond distances are indicated and shown in yellow (Koutmos et al., 2010). 
Yadav et al. (2012) observed that while the substitution of either threonine residue (257 or 260) of the Gly-rich loop resulted in impaired heme binding, neither interact directly with this cofactor. The authors proposed that $\alpha$-helix 8 , which extends from the heme cofactor to the PLP cofactor may be involve in bi-directional communication through the interactions of the Gly-rich loop at the N-terminal end of the helix and residue R266, which interacts with the thiolate atom of C52, one of the axial ligands of the heme iron (Figure 4.5) (Weeks et al., 2009; Yadav et al., 2012). The results of this study suggest that residue T257 may participate in this $\alpha$-helix-8-mediated communication as its proximity to the phosphate moiety of PLP allows the residue to function as a signal transducer between the cofactor sites.

Previous work has focused on the Thr residues of the Gly-rich loop while the Gly residues have not been investigated (Yadav et al., 2012). The replacement of G259 with aspartate or methionine results in a similar reduction in catalytic efficiency as the T257M variant despite their more pronounced effect on the electronic environment of heme than htCBS-T257M. The Soret peak of both G259-substitution variants has blue-shifted $25 \mathrm{~nm}$ to $405 \mathrm{~nm}$ (Figure 3.5B). This is accompanied by a broadening of the Soret peak and a 34-fold reduction in the $\mathrm{A}_{430 / 280}$ ratio. Ojha and collegues (2000) demonstrated the use of mercury chloride, a thiol chelator, in broadening the Soret peak of hfCBS as well as a peak shift to $\sim 395 \mathrm{~nm}$. X-Ray absorption data indicates a loss of C52 without a replacement (Ojha et al., 2000). The broadening of the G259D/M Soret peaks and their reported blue-shift may indicate the occurrence of a similar effect. The insertion of bulkier side chains at position 259 may shift the positioning of PLP and $\alpha$-helix 8 , resulting in loss of the thiolate heme ligand as a result of the C52-R266 interaction. 


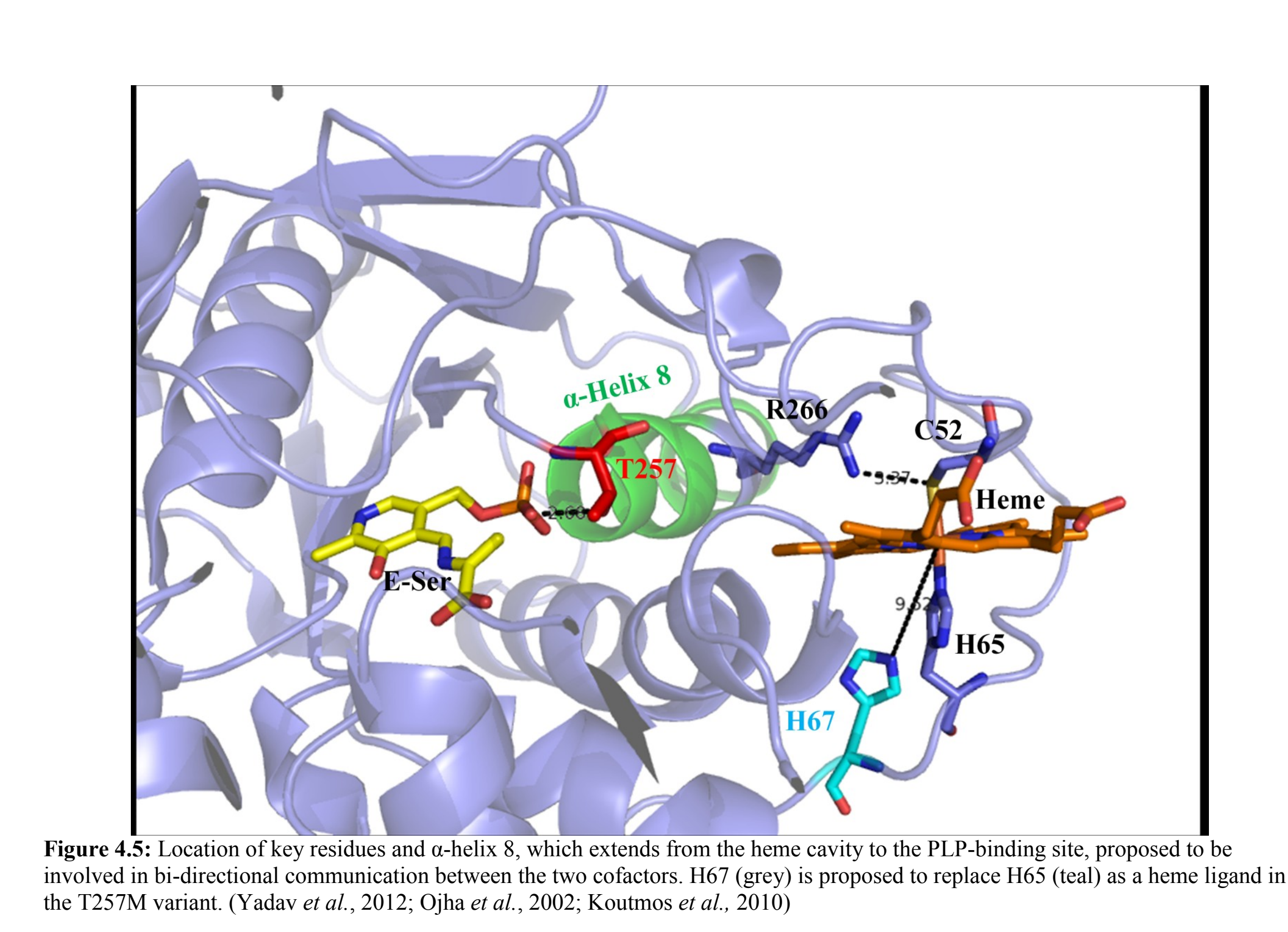




\subsubsection{Residue $S 349$}

Residue S349 is situated in the C-terminal segment of the catalytic core of CBS. The hydroxyl group of the S349 side chain participates in positioning and modulating the electronic properties of the PLP cofactor via a hydrogen bond with the N1 nitrogen of the pyridine ring (Figure 4.6) (Meier et al., 2001; Koutmos et al., 2010). Unlike the other residues examined in this study, which are conserved in all known CBS enzymes, S349 is conserved more broadly, across the members of the fold-type II group of PLP-dependent enzymes. This is suggestive of its importance to the family's ability to catalyze $\beta$ replacement reactions.

The introduction of an asparagine residue at this position interferes with the enzyme's ability to catalyze its physiological reaction, as demonstrated by the 14 -fold reduction in $k_{\text {cat }}{ }^{L-S e r}$. Replacement of the corresponding S289 of the model ytCBS enzyme with alanine results in a 20 -fold decline in $k_{c a t}$, while substitution with aspartate, present in this position in many fold-type I PLP-dependent enzymes, leads to a loss of $\beta$ replacement activity (Quazi and Aitken, 2009). While there was no change in the overall spectral profile of htCBS-S349N, the $A_{430 / 280}$ ratio is decreased 1.5 -fold and is the lowest among the htCBS variants that maintained a similar absorbance spectrum to the wild-type enzyme. The altered interaction between the substituted asparagine residue and the pyridine ring may have been communicated to heme, via $\alpha$-helix 8 , thereby reducing heme binding or altering the intensity of the Soret peak. Although the S349N variant is not $\mathrm{B}_{6}$-responsive, it is interesting to note that substitution of S349 with asparagine, which is of similar size to aspartate, is accommodated in the context of htCBS, in contrast with the inactive ytCBS-S289D variant (Urreizti et al., 2003; Quazi and Aitken, 2009). 


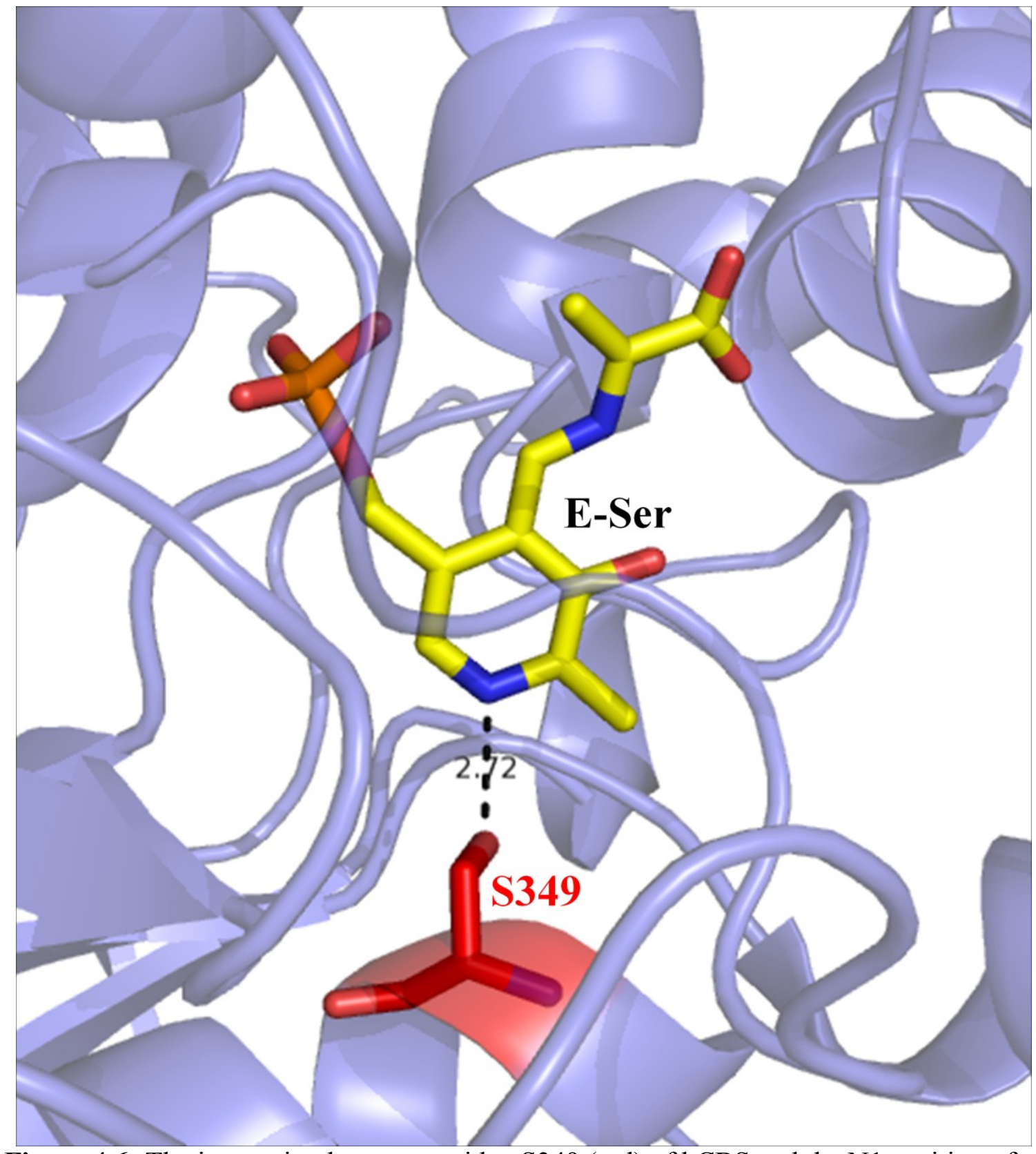

Figure 4.6: The interaction between residue S349 (red) of hCBS and the N1 position of the PLP cofactor (E-Ser; yellow). The proposed hydrogen bond and hydrogen bond distance is indicated in yellow (Lodha et al., 2010; Meier et al., 2001; Koutmos et al., 2010). 


\subsubsection{Residues G305 \& G307}

Residues G305 and G307 are located at the mouth of the active site (Figure 4.7). G305 aids in the orientation of PLP through Van der Waals interactions with the re face of pyridine ring. Located at the bottom of the active site opening, G307 is situated in a tightly packed pocket with P282, S285 and T301 (Meier et al., 2003). Meier et al. (2003) hypothesized that the G305R substitution likely would affect cofactor binding because of the need to accommodate the large, positively charged side chain of arginine. The 11-fold reduction in $k_{\text {cat }}^{L-S e r}$ for htCBS-G305R imply that the cofactor remains in a catalyticallyproductive position within the active site, suggesting that the loop of residues including G305 can adopt an alternate conformation to accommodate the bulky arginine side chain (Kraus et al., 1999). Remarkably, a patient heterozygous for both the I278T and G305R variants was found to be $\mathrm{B}_{6}$-responsive suggesting that the treatment may be sufficient for G305R homozygous patients (Sperandeo et al., 1996).

The insertion of a serine residue at position 307 is believed to cause a protrusion within the tightly packed group of residues located at the bottom of the active site entrance. The small size of G307 allows space for other bulky residues within its vicinity such as P282 and T301 and to accommodate L-Hcys binding. Modeling of the G307S substitution in hCBS suggested the formation of a new hydrogen bond between the hydroxyl groups of the side chains of S307 and T301, causes a conformational change,resulting in the loss of hydrogen bonds between the phosphate moiety of PLP and residues G256 and T257. This may explain the reduced activity and unresponsiveness to vitamin $\mathrm{B}_{6}$ observed for the common G307S allele (Lodha et al., 2009; Kraus et al., 1999). While the corresponding substitution (ytCBS-G247S) resulted in a lack of $\beta$ replacement activity in the model yeast enzyme (Lodha et al., 2009), the truncated human 
counterpart exhibits detectable activity. Comparison of the htCBS-S349N and G307S substitutions with the corresponding substitutions in the model yeast enzyme illustrate the subtle structure-function relationships that underlie enzyme function, as differences are observed despite the $47 \%$ amino acid sequence identity shared by the catalytic cores of the human and yeast CBS enzymes (Jhee et al., 2000a; Jhee et al., 2000b). 


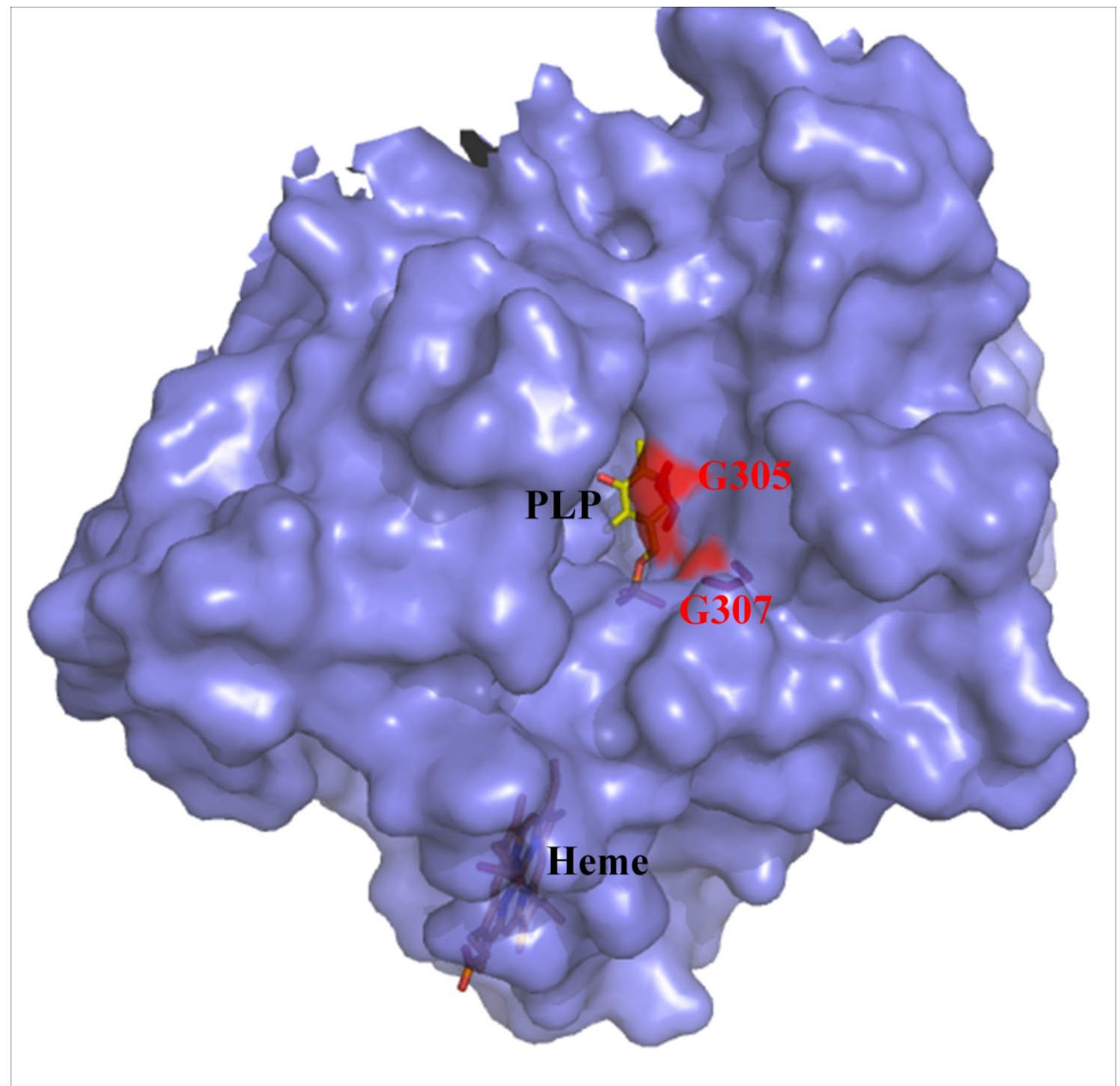

Figure 4.7: Position of G305 and G307 (red) at the mouth of the hCBS active site. The PLP (yellow) and heme (orange) cofactors are highlighted (Meier et al., 2001). 


\section{CONCLUSION}

This study focused on the exploration of the structure-function relationships that underlie the interaction networks and the effects of a selection of homocystinuriaassociated mutations in the active site of CBS. The role of active-site residues D319, S323 and Y324 in positioning of the pyridoxal 5'-phosphate cofactor was investigated in the model yeast CBS enzyme. In the context of yfCBS, the separate removal of the sidechain hydrogen bonding capacity of residues S323 and Y324 resulted in minor changes to enzymatic function suggesting that neither is a key determinant of cofactor orientation or active-site architecture. The inactivity of the D319A variant may be due to a shift in the orientation of PLP. Removal of the carboxylate moiety of D319 from the active-site hydrogen bonding network may lead to a repositioning of N84 or a change in the strength of the N84-PLP (O3') interaction.

The effect of a set of 11 homocystinuria-associated substitutions, located within the active-site, on the kinetic parameters of human CBS was also investigated. Residue G148 allows for the flexibility of the mobile loop, essential for proper transitions between the open and closed active site conformations. Residues E176, G307 and K384 are responsible for maintaining proper active site architecture. Residues in proximity to the phosphate moiety of PLP (T257, G259) are vital to the effective binding of PLP along with G305 and S349 in addition to the bi-directional communication between the heme and PLP cofactors. This is the first step to a better understanding of the molecular mechanism of homocystinuria. As there are $>160$ reported mutations associated with the disorder that span all three major domains of hCBS, gaining insight into the structurefunction relationship can help postulate novel therapeutics for patients with homocystinuria. 


\section{REFERENCES}

Abe, K., \& Kimura, H. (1996). The possible role of hydrogen sulfide as an endogenous neuromodulator. Journal of Neuroscience, 16: 1066-1071.

Aitken, S.M., \& Kirsch, J.F. (2003). Kinetics of the yeast cystathionine ß-synthase forward and reverse reactions: Continuous assays and the equilibrium constant for the reaction. Biochemistry, 42: 571-578.

Aitken, S.M., \& Kirsch, J.F. (2005). The enzymology of cystathionine biosynthesis: Strategies for the control of substrate and reaction specificity. Archives of Biochemistry and Biophysics, 433: 166-175.

Aitken, S.M., Lodha, P.H., \& Morneau, D.J.K. (2011). The enzymes of the transsulfuration pathways: Active-site characterizations. Biochimica Et Biophysica Acta Proteins and Proteomics, 1814: 1511-1517.

Aitken, S.M., \& Kirsch, J.F. (2004). Role of active-site residues Thr81, Ser82, Thr85, Gln157, and Tyr158 inYeast cystathionine $\beta$-synthase catalysis and reaction specificity. Biochemistry, 43: 1963-1971.

Atmaca, G. (2004). Antioxidant effects of sulfur-containing amino acids. Yonsei Medical Journal, 45: 776-788.

Banerjee, R., Evande, R., Kabil, Ö., Ojha, S., \& Taoka, S. (2003). Reaction mechanism and regulation of cystathionine $\beta$-synthase. Biochimica Et Biophysica Acta-Proteins and Proteomics, 1647: 30-35.

Banerjee, R., \& Zou, C-G. (2005). Redox regulation and reaction mechanism of human cystathionine- $\beta-$ synthase: A PLP-dependent hemesensor protein. Archives of Biochemistry and Biophysics, 433: 144-156.

Bateman, A. (1997). The structure of a domain common to archaebacteria and the homocystinuria disease protein. Trends in Biochemical Sciences, 22: 12-13.

Belew, M.S., Quazi, F.I., Willmore, W.G., \& Aitken, S.M. (2009). Kinetic characterization of recombinant human cystathionine beta-synthase purified from E. coli. Protein Expression and Purification, 64: 139-145.

Bonaa, K.H., Njolstad, I., Ueland, P.M., Schirmer, H., Tverdal, A., Steigen, T., Wang, H., Nordrehaug, J.E., Arnesen, E., Rasmussen, K. \& the NORVIT Trial Investigators. (2006). Homocysteine lowering and cardiovascular events after acute myocardial infarction. The New England Journal of Medicine, 354: 1578-1588. 
Brosnan, J.T., \& Brosnan, M.E. (2006). The sulfur-containing amino acids: An overview. Journal of Nutrition, 136: 1636S-1640S.

Burkhard, P., Tai, C.H., Ristroph, C.M., Cook, P.F., \& Jansonius, J.N. (1999). Ligand binding induces a large conformational change in O-acetylserine sulfhydrylase from salmonella typhimurium. Journal of Molecular Biology, 291: 941-953.

Carballal, S., Cuevasanta, E., Marmisolle, I., Kabil, O., Gherasim, C., Ballou, D.P., Banerjee, R. \& Alvarez, B. (2013). Kinetics of reversible reductive carbonylation of heme in human cystathionine B-synthase. Biochemistry, 52: 4553-4562.

Carson, N.A., \& Neill, D.W. (1962). Metabolic abnormalities detected in a survey of mentally backward individuals in northern ireland. Archives of Disease in Childhood, 37: 505-513.

Chang, Z., \& Vining, L.C. (2002). Biosynthesis of sulfur-containing amino acids in streptomyces venezuelae ISP5230: Roles for cystathionine beta-synthase and transsulfuration. Microbiology, 148: 2135-2147.

Christen, P., \& Mehta, P.K. (2001). From cofactor to enzymes. the molecular evolution of pyridoxal-s'-pliosphate-dependent enzymes. Chemical Records, 1: 436-447.

Clarke, R., Daly, L., Robinson, K., Naughten, E., Cahalane, S., Fowler, B., \& Graham, I. (1991). Hyperhomocysteinemia: An independent risk factor for vascular disease. The New England Journal of Medicine, 324: 1149-1155.

Clarke, R., Smith, A.D., Jobst, K.A., Refsum, H., Sutton, L., \& Ueland, P.M. (1998). Folate, vitamin B12, and serum total homocysteine levels in confirmed alzheimer disease. Archives of Neurology, 55: 1449-1455.

Clayton, P.T. (2006). B6-responsive disorders: A model of vitamin dependency. Journal of Inherited Metabolic Disease, 29: 317-326.

Cruysberg, J.R.M., Boers, G.H.J., Trijbels, J.M.F., \& Deutman, A.F. (1996). Delay in diagnosis of homocystinuria: Retrospective study of consecutive patients. British Medical Journal, 313: 1037-1040.

Daubner, S.C., \& Matthews, R.G. (1982). Purification and properties of methylenetetrahydrofolate reductase from pig liver. The Journal of Biological Chemistry, 257: 140-145. 
Dunathan, H.C. (1966). Conformation and reaction specificity in pyridoxal phosphate enzymes. Proceedings of the National Academy of Sciences of the United States of America, 55: 712-716.

Eliot, A.C., \& Kirsch, J.F. (2004). Pyridoxal phosphate enzymes: Mechanistic, structural, and evolutionary considerations. Annual Review of Biochemistry, 73: 383-415.

Ereno-Orbea, J., Majtan, T., Oyenarte, I., Kraus, J.P., \& Martinez-Cruz, L.A. (2013). Structural basis of regulation and oligomerization of human cystathionine beta-synthase, the central enzyme of transsulfuration. Proceedings of the National Academy of Sciences of the United States of America, 110: E3790-9.

Ereno-Orbea, J., Oyenarte, I., \& Martinez-Cruz, L.A. (2013). CBS domains: Ligand binding sites and conformational variability. Archives of Biochemistry and Biophysics, 540: 70-81.

Evande, R., Blom, H., Boers, G.H.J., \& Banerjee, R. (2002). Alleviation of intrasteric inhibition by the pathogenic activation domain mutation, D444N, in human cystathionine ß-synthase. Biochemistry, 41: 11832-11837.

Evande, R., Ojha, S., \& Banerjee, R. (2004). Visualization of PLP-bound intermediates in hemeless variants of human cystathionine $\beta$-synthase: Evidence that lysine 119 is a general base. Archives of Biochemistry and Biophysics, 427: 188-196.

Evans, C.L. (1967). The toxicity of hydrogen sulphide and other sulphides. Quarterly Journal of Experimental Physiology and Cognate Medical Sciences, 52: 231-248.

Farsi, A., Lodha, P. H., Skanes, J.E., Los, H., Kalidindi, N., \& Aitken, S.M. (2009). Interconversion of a pair of active-site residues in escherichia coli cystathionine gammasynthase, E. coli cystathionine beta-lyase, and saccharomyces cerevisiae cystathionine gamma-lyase and development of tools for the investigation of their mechanisms and reaction specificity. Biochemistry and Cell Biology = Biochimie Et Biologie Cellulaire, 87: 445-457.

Finkelstein, J.D. (1998). The metabolism of homocysteine: Pathways and regulation. European Journal of Pediatrics, Supplement, 157: S40-S44.

Finkelstein, J.D., Kyle, W.E., Martin, J.J., \& Pick, A.M. (1975). Activation of cystathionine synthase by adenosylmethionine and adenosylethionine. Biochemical and Biophysical Research Communications, 66: 81-87. 
Finkelstein, J.D., \& Martin, J.J. (1984). Inactivation of betaine-homocysteine methyltransferase by adenosylmethionine and adenosylethionine. Biochemical and Biophysical Research Communications, 118: 14-19.

Froger, A., \& Hall, J.E. (2007). Transformation of plasmid DNA into E. coli using the heat shock method. Journal of Visualized Experiments : JoVE, 6: 253

Gerritsen, T., Vaughn, J.G., \& Waisman, H.A. (1962). The identification of homocystine in the urine. Biochemical and Biophysical Research Communications, 9: 493-496.

Harris, E.D., \& Sjoerdsma, A. (1966). Collagen profile in various clinical conditions. Lancet, 2: 707-711.

Hayashi, H. (1995). Pyridoxal enzymes: Mechanistic diversity and uniformity. Journal of Biochemistry, 118: 463-473.

Higuchi, R., Krummel, B., \& Saiki, R.K. (1988). A general method of in vitro preparation and specific mutagenesis of DNA fragments: Study of protein and DNA interactions. Nucleic Acids Research, 16: 7351-7367.

Hnizda, A., Majtan, T., Liu, L., Pey, A.L., Carpenter, J.F., Kodicek, M., Kozich, V. \& Kraus, J.P. (2012). Conformational properties of nine purified cystathionine betasynthase mutants. Biochemistry, 51: 4755-4763.

Hosoki, R., Matsuki, N., \& Kimura, H. (1997). The possible role of hydrogen sulfide as an endogenous smooth muscle relaxant in synergy with nitric oxide. Biochemical and Biophysical Research Communications, 237: 527-531.

Jackson, S.H. (1973). The reaction of homocysteine with aldehyde: An explanation of the collagen defects in homocystinuria. Clinica Chimica Acta, 45: 215-217.

Jakubowski, H. (2008). The pathophysiological hypothesis of homocysteine thiolactonemediated vascular disease. Journal of Physiology and Pharmacology, 59: 155-167.

Janošík, M., Kery, V., Gaustadnes, M., Maclean, K.N., \& Kraus, J.P. (2001). Regulation of human cystathionine $\beta$-synthase by S-adenosyl-L-methionine: Evidence for two catalytically active conformations involving an autoinhibitory domain in the C-terminal region. Biochemistry, 40: 10625-10633.

Janosik, M., Oliveriusova, J., Janosikova, B., Sokolova, J., Kraus, E., Kraus, J.P., \& Kozich, V. (2001). Impaired heme binding and aggregation of mutant cystathionine betasynthase subunits in homocystinuria. American Journal of Human Genetics, 68: 15061513. 
Jhee, K-H., McPhie, P., \& Miles, E.W. (2000a). Domain architecture of the hemeindependent yeast cystathionine $\beta$-synthase provides insights into mechanisms of catalysis and regulation. Biochemistry, 39: 10548-10556.

Jhee, K-H., McPhie, P., \& Miles, E.W. (2000b). Yeast cystathionine ß-synthase is a pyridoxal phosphate enzyme but, unlike the human enzyme, is not a heme protein. Journal of Biological Chemistry, 275: 11541-11544.

Jhee, K-H., Niks, D., McPhie, P., Dunn, M.F., \& Miles, E.W. (2001). The reaction of yeast cystathionine $\beta$-synthase is rate-limited by the conversion of aminoacrylate to cystathionine. Biochemistry, 40: 10873-10880.

Kabil, H., Kabil, O., Banerjee, R., Harshman, L.G., \& Pletcher, S.D. (2011). Increased transsulfuration mediates longevity and dietary restriction in drosophila. Proceedings of the National Academy of Sciences of the United States of America, 108: 16831-16836.

Kang, A.H., \& Trelstad, R.L. (1973). A collagen defect in homocystinuria. Journal of Clinical Investigation, 52:, 2571-2578.

Kerrin, D., Eaton, D.M., Livingston, J., Henderson, M., \& Smith, M. (1996). Homocystinuria presenting with sagittal sinus thrombosis in infancy. Journal of Child Neurology, 11: 70-71.

Kery, V., Bukovska, G., \& Kraus, J.P. (1994). Transsulfuration depends on heme in addition to pyridoxal 5'-phosphate. cystathionine B-synthase is a heme protein. Journal of Biological Chemistry, 269: 25283-25288.

Kery, V., Poneleit, L., \& Kraus, J.P. (1998). Trypsin cleavage of human cystathionine Bsynthase into an evolutionarily conserved active core: Structural and functional consequences. Archives of Biochemistry and Biophysics, 355: 222-232.

Kery, V., Poneleit, L., Meyer, J.D., Manning, M.C., \& Kraus, J.P. (1999). Binding of pyridoxal 5'-phosphate to the heme protein human cystathionine betasynthase. Biochemistry, 38: 2716-2724.

Kimura, H. (2000). Hydrogen sulfide induces cyclic AMP and modulates the NMDA receptor. Biochemical and Biophysical Research Communications, 267: 129-133.

Kimura, H. (2013). Physiological role of hydrogen sulfide and polysulfide in the central nervous system. Neurochemistry International, 63: 492-497.

Kimura, Y., \& Kimura, H. (2004). Hydrogen sulfide protects neurons from oxidative stress. The FASEB Journal, 18: 1165-1167 
Koutmos, M., Kabil, O., Smith, J.L., \& Banerjee, R. (2010). Structural basis for substrate activation and regulation by cystathionine beta-synthase (CBS) domains in cystathionine B-synthase. Proceedings of the National Academy of Sciences of the United States of America, 107: 20958-20963.

Kožich, V., Sokolová, J., Klatovská, V., Krijt, J., Janošík, M., Jelínek, K., \& Kraus, J.P. (2010). Cystathionine B-synthase mutations: Effect of mutation topology on folding and activity. Human Mutation, 31: 809-819.

Kraus, J.P., Janosik, M., Kožich, V., Mandell, R., Shih, V., Sperandeo, M.P., Sebastio, G., de Franchis, R., Andria, G., Kluijtmans, L.A.J., Blom, H., Boers, G.H.J., Gordon, R.B., Kamoun, P., Tsai, M.Y., Kruger, W.D., Koch, H.G., Ohura, T. \& Gaustadnes, M. (1999). Cystathionine ß-synthase mutations in homocystinuria. Human Mutation, 13: $362-375$.

Kraus, J.P., Oliveriusová, J., Sokolová, J., Kraus, E., Vlcek, C., De Franchis, R., Maclean, K.N., Boa, L., Bukovska, G., Patterson, D., Paces, V., Ansorge, W. \& Kožich, V. (1998). The human cystathionine $\beta$-synthase (CBS) gene: Complete sequence, alternative splicing, and polymorphisms. Genomics, 52: 312-324.

Li, J. G., Chu, J., Barrero, C., Merali, S., \& Pratico, D. (2014). Homocysteine exacerbates beta-amyloid pathology, tau pathology, and cognitive deficit in a mouse model of alzheimer disease with plaques and tangles. Annals of Neurology, 75: 851-863.

Lipton, S.A., Kim, W-K., Choi, Y-B., Kumar, S., D'Emilia, D.M., Rayudu, P.V., Arnell, D.R. \& Stamler, J.S. (1997). Neurotoxicity associated with dual actions of homocysteine at the N- methyl-D-aspartate receptor. Proceedings of the National Academy of Sciences of the United States of America, 94: 5923-5928.

Lodha, P.H., Hopwood, E.M.S., Manders, A.L., \& Aitken, S.M. (2010). Residue N84 of yeast cystathionine $\beta$-synthase is a determinant of reaction specificity. Biochimica Et Biophysica Acta - Proteins and Proteomics, 1804: 1424-1431.

Lodha, P.H., Shadnia, H., Woodhouse, C.M., Wright, J.S., \& Aitken, S.M. (2009). Investigation of residues Lys112, Glu136, His138, Gly247, Tyr248, and Asp249 in the active site of yeast cystathionine $\beta$-synthase. Biochemistry and Cell Biology, 87: 531540 .

MacLean, K.N., Janošík, M., Oliveriusová, J., Kery, V., \& Kraus, J.P. (2000). Transsulfuration in saccharomyces cerevisiae is not dependent on heme: Purification and characterization of recombinant yeast cystathionine $\beta$-synthase. Journal of Inorganic Biochemistry, 81: 161-171. 
Majors, A.K., Sengupta, S., Willard, B., Kinter, M.T., Pyeritz, R.E., \& Jacobsen, D.W. (2002). Homocysteine binds to human plasma fibronectin and inhibits its interaction with fibrin. Arteriosclerosis, Thrombosis, and Vascular Biology, 22: 1354-1359.

Majtan, T., Liu, L., Carpenter, J.F., \& Kraus, J.P. (2010). Rescue of cystathionine betasynthase (CBS) mutants with chemical chaperones: Purification and characterization of eight CBS mutant enzymes. The Journal of Biological Chemistry, 285: 15866-15873.

Majtan, T., Freeman, K.M., Smith, A.T., Burstyn, J.N., \& Kraus, J.P. (2011). Purification and characterization of cystathionine $\beta$-synthase bearing a cobalt protoporphyrin. Archives of Biochemistry and Biophysics, 508: 25-30

Majtan, T., Singh, L.R., Wang, L., Kruger, W.D., \& Kraus, J.P. (2008). Active cystathionine $\beta$-synthase can be expressed in heme-free systems in the presence of metalsubstituted porphyrins or a chemical chaperone. Journal of Biological Chemistry, 283: 34588-34595.

Meier, M., Janosik, M., Kery, V., Kraus, J.P., \& Burkhard, P. (2001). Structure of human cystathionine $\beta$-synthase: A unique pyridoxal 5'-phosphate-dependent heme protein. EMBO Journal, 20: 3910-3916.

Meier, M., Oliveriusova, J., Kraus, J.P., \& Burkhard, P. (2003). Structural insights into mutations of cystathionine ß-synthase. Biochimica Et Biophysica Acta - Proteins and Proteomics, 1647: 206-213.

Miles, E.W., \& Kraus, J.P. (2004). Cystathionine ß-synthase: Structure, function, regulation, and location of homocystinuria-causing mutations. Journal of Biological Chemistry, 279: 29871-29874.

Morrow, G., \& Barness, L.A. (1972). Combined vitamin responsiveness in homocystinuria. The Journal of Pediatrics, 81: 946-954.

Mudd, S., Levy, H., \& Kraus, J. (2001). Disorders of transsulfuration. In C. Scriver, A. Beaudet, W. Sly, D. Valle, B. Childs, K. Kinzler \& B. Vogelstein (Eds.), The metabolic \& molecular bases of inherited disease (8th Ed., pp. 2007-2056). New York: McGrawHill Companies Inc.

Mudd, H.S., Finkelstein, J.D., Irreverre, F., \& Laster, L. (1964). Homocystinuria: An enzymatic defect. Science, 143: 1443-1445. 
Mudd, S.H., Skovby, F., Levy, H.L., Pettigrew, K.D., Wilcken, B., Pyeritz, R.E, Andria, G., Boers, G.H.J., Bromberg, I.L., Cerone, R., Fowler, B., Grobe, H., Schmidt, H. \& Schweitzer, L. (1985). The natural history of homocystinuria due to cystathionine betasynthase deficiency. American Journal of Human Genetics, 37: 1-31.

Munke, M., Kraus, J.P., Ohura, T., \& Francke, U. (1988). The gene for cystathionine ßsynthase (CBS) maps to the subtelomeric region on human chromosome $21 \mathrm{q}$ and to proximal mouse chromosome 17. American Journal of Human Genetics, 42: 550-559.

Naughten, E.R., Yap, S., \& Mayne, P.D. (1998). Newborn screening for homocystinuria: Irish and world experience. European Journal of Pediatrics, Supplement, 157: S84-S87.

Ojha, S., Hwang, J., Kabil, O., Penner-Hahn, J. E., \& Banerjee, R. (2000). Characterization of the heme in human cystathionine $\beta$-synthase by X-ray absorption and electron paramagnetic resonance spectroscopies. Biochemistry, 39: 10542-10547.

Ojha, S., Wu, J., LoBrutto, R., \& Banerjee, R. (2002). Effects of heme ligand mutations including a pathogenic variant, H65R, on the properties of human cystathionine Bsynthase. Biochemistry, 41: 4649-4654.

Oyenarte, I., Majtan, T., Ereno, J., Corral-Rodriguez, M.A., Kraus, J.P., \& MartinezCruz, L.A. (2012). Purification, crystallization and preliminary crystallographic analysis of human cystathionine beta-synthase. Acta Crystallographica.Section F, Structural Biology and Crystallization Communications, 68: 1318-1322.

Ozkan, Y., Ozkan, E., \& Simsek, B. (2002). Plasma total homocysteine and cysteine levels as cardiovascular risk factors in coronary heart disease. International Journal of Cardiology, 82: 269-277.

Pagon, R.A., Adam, M.P., Bird, T.D., Dolan, C.R., Fong, C., Stephens, K., Picker, J.D. \& Levy, H.L. (2011). Homocystinuria caused by cystathionine beta-synthase deficiency.

Pasternak, J.J. (2005). An introduction to human molecular genetics: Mechanisms of inherited diseases (2nd Ed.). Hoboken, NJ: John Wiley \& Sons Inc. 1-8.

Percudani, R., \& Peracchi, A. (2003). A genomic overview of pyridoxal-phosphatedependent enzymes. EMBO Reports, 4: 850-854.

Perna, A.F., Ingrosso, D., Lombardi, C., Acanfora, F., Satta, E., Cesare, C.M., Violetti, E., Romano, M.M. \& De Santo, N.G. (2003). Possible mechanisms of homocysteine toxicity. Kidney International, 63: S137-S140. 
Pey, A.L., Majtan, T., Sanchez-Ruiz, J.M., \& Kraus, J.P. (2013). Human cystathionine beta-synthase (CBS) contains two classes of binding sites for S-adenosylmethionine (SAM): Complex regulation of CBS activity and stability by SAM. The Biochemical Journal, 449: 109-121.

Quazi, F., \& Aitken, S.M. (2009). Characterization of the S289A,D mutants of yeast cystathionine beta-synthase. Biochimica Et Biophysica Acta, 1794: 892-897.

Sakai, L.Y. (1990). Disulphide bonds crosslink molecules of fibrillin in the connective tissue space, In A. Tamburro \& J.M. Davidson (Eds.), Elastin: Chemical and Biological Aspects (pp. 213-277).

Saposnik, G., Ray, J.G., Sheridan, P., McQueen, M., Lonn, E., \& Heart Outcomes Prevention Evaluation 2 Investigators. (2009). Homocysteine-lowering therapy and stroke risk, severity, and disability: Additional findings from the HOPE 2 trial. Stroke, 40: 1365-1372.

Schneider, G., Käck, H., \& Lindqvist, Y. (2000). The manifold of vitamin B6 dependent enzymes. Structure, 8: R1-R6.

Scott, J.W., Hawley, S.A., Green, K.A., Anis, M., Stewart, G., Scullion, G.A., Norman, D.G. \& Hardie, D.G. (2004). CBS domains form energy-sensing modules whose binding of adenosine ligands is disrupted by disease mutations. The Journal of Clinical Investigation, 113: 274-284.

Scriver, C.R. (2008). Garrod's croonian lectures (1908) and the charter 'inborn errors of metabolism': Albinism, alkaptonuria, cystinuria, and pentosuria at age 100 in 2008. Journal of Inherited Metabolic Disease, 31: 580-598.

Singh, R.H., Kruger, W.D., Wang, L., Pasquali, M., \& Elsas, L.J. (2004). Cystathionine ß-synthase deficiency: Effects, of betaine supplementation after methioninie restriction in B6-nonresponsive homocystinuria. Genetics in Medicine, 6: 90-95.

Singh, S., \& Banerjee, R. (2011). PLP-dependent H 2S biogenesis. Biochimica Et Biophysica Acta - Proteins and Proteomics, 1814: 1518-1527.

Singh, S., Madzelan, P., Stasser, J., Weeks, C.L., Becker, D., Spiro, T.G., Penner-Hahn, J. \& Banerjee, R. (2009a). Modulation of the heme electronic structure and cystathionine B-synthase activity by second coordination sphere ligands: The role of heme ligand switching in redox regulation. Journal of Inorganic Biochemistry, 103: 689-697. 
Singh, S., Padovani, D., Leslie, R.A., Chiku, T., \& Banerjee, R. (2009b). Relative contributions of cystathionine $\beta$-synthase and $\gamma$-cystathionase to $\mathrm{H} 2 \mathrm{~S}$ biogenesis via alternative trans-sulfuration reactions. Journal of Biological Chemistry, 284: 2245722466.

Smith, A.T., Su, Y., Stevens, D.J., Majtan, T., Kraus, J.P., \& Burstyn, J.N. (2012). Effect of the disease-causing R266K mutation on the heme and PLP environments of human cystathionine $\beta$-synthase. Biochemistry, 51: 6360-6370.

Sperandeo, M.P., de Franchis, R., Andria, G., \& Sebastio, G. (1996). A 68-bp insertion found in a homocystinuric patient is a common variant and is skipped by alternative splicing of the cystathionine beta-synthase mRNA. American Journal of Human Genetics, 59: 1391-1393.

Stamler, J.S., \& Slivka, A. (1996). Biological chemistry of thiols in the vasculature and in vascular-related disease. Nutrition Reviews, 54: 1-30.

Steegborn, C., Clausen, T., Sondermann, P., Jacob, U., Worbs, M., Marinkovic, S., Huber, R. \& Wahl, M.C. (1999). Kinetics and inhibition of recombinant human cystathionine $\gamma$-lyase: Toward the Rational Control of Transsulfuration. Journal of Biological Chemistry, 274: 12675-12684

Stipanuk, M.H. (2004). Sulfur amino acid metabolism: Pathways for production and removal of homocysteine and cysteine. Annual Reviews of Nutrition, 24: 539-577.

Taoka, S., \& Banerjee, R. (2001). Characterization of NO binding to human cystathionine B-synthase: Possible implications of the effects of $\mathrm{CO}$ and $\mathrm{NO}$ binding to the human enzyme. Journal of Inorganic Biochemistry, 87: 245-251.

Taoka, S., Ohja, S., Shan, X., Kruger, W.D., \& Banerjee, R. (1998). Evidence for hememediated redox regulation of human cystathionine beta-synthase activity. The Journal of Biological Chemistry, 273: 25179-25184.

Urreizti, R., Balcells, S., Rodes, M., Vilarinho, L., Baldellou, A., Couce, M.L., Munoz, C., Campistol, J., Pinto, X., Vilaseca, M.A. \& Grinberg, D. (2003). Spectrum of CBS mutations in 16 homocystinuric patients from the iberian peninsula: High prevalence of T191M and absence of I278T or G307S. Human Mutation, 22: 103-111.

Weeks, C.L., Singh, S., Madzelan, P., Banerjee, R., \& Spiro, T.G. (2009). Heme regulation of human cystathionine $\beta$-synthase activity: Insights from fluorescence and raman spectroscopy. Journal of the American Chemical Society, 131: 12809-12816. 
Wu, G. (2009). Amino acids: Metabolism, functions, and nutrition. Amino Acids, 37: 117.

Wu, G., Fang, Y.Z., Yang, S., Lupton, J.R., \& Turner, N.D. (2004). Glutathione metabolism and its implications for health. The Journal of Nutrition, 134: 489-492.

Yadav, P.K., \& Banerjee, R. (2012). Detection of reaction intermediates during human cystathionine B-synthase-monitored turnover and $\mathrm{H} 2 \mathrm{~S}$ production. Journal of Biological Chemistry, 287: 43464-43471.

Yadav, P.K., Xie, P., \& Banerjee, R. (2012). Allosteric communication between the pyridoxal 5'-phosphate (PLP) and heme sites in the H2S generator human cystathionine $\beta$-synthase. Journal of Biological Chemistry, 287: 37611-37620.

Yamanishi, M., Kabil, O., Sen, S., \& Banerjee, R. (2006). Structural insights into pathogenic mutations in heme-dependent cystathionine-ß-synthase. Journal of Inorganic Biochemistry, 100: 1988-1995.

Yap, S. (2012). Classical homocystinuria: Newborn screening with early treatment effectively prevents complications. Hamdan Medical Journal, 5: 351-362.

Zhang, C., Chi, F.L., Xie, T.H., \& Zhou, Y.H. (2013). Effect of B-vitamin supplementation on stroke: A meta-analysis of randomized controlled trials. PloS One, 8:, e81577.

Zschocke, J., Kebbewar, M., Gan-Schreier, H., Fischer, C., Fang-Hoffmann, J., Wilrich, J., Abdoh, G., Ben-Omran, T., Shahbek, N., Lindner, M., Al Rifai, H., Al Khal, A.L. \& Hoffmann, G.F. (2009). Molecular neonatal screening for homocystinuria in the qatari population. Human Mutation, 30: 1021-1022. 